A N N A L E S Annales de Bretagne et des Pays de l'Ouest

\title{
Sébastien de La Haye de Silz : jeunesse tumultueuse et années d'enfermement
}

Sébastien de la Haye de Silz's tumultuous youth and years of imprisonment

\section{Camille Le Masne et Pierre Le Masne}

\section{(2) OpenEdition \\ 1 Journals}

Édition électronique

URL : http://journals.openedition.org/abpo/3438

DOI : $10.4000 / a b p o .3438$

ISBN : 978-2-7535-5365-1

ISSN : $2108-6443$

Éditeur

Presses universitaires de Rennes

Édition imprimée

Date de publication : 30 décembre 2016

Pagination : 133-161

ISBN : 978-2-7535-5363-7

ISSN : $0399-0826$

\section{Référence électronique}

Camille Le Masne et Pierre Le Masne, « Sébastien de La Haye de Silz : jeunesse tumultueuse et années d'enfermement », Annales de Bretagne et des Pays de l'Ouest [En ligne], 123-4 | 2016, mis en ligne le 30 décembre 2018, consulté le 03 janvier 2020. URL : http://journals.openedition.org/abpo/ 3438 ; DOI : 10.4000/abpo.3438 


\title{
Sébastien de La Haye de Silz : jeunesse tumultueuse et années d'enfermement
}

\author{
Camille LE MASNE \\ Docteur en géographie, chercheur au CESSMA (UMR 245)
}

Pierre LE MASNE ${ }^{1}$

Maître de conférences HDR en sciences économiques à l'université de Poitiers, chercheur au CRIEF (EA 2249)

Sébastien de La Haye de Silz a été le chef de la chouannerie du Morbihan en 1794-1795 et a su s'imposer à ce titre aux guerriers redoutables qu'il dirigeait, Cadoudal, Guillemot, Jean Jan. Le nom de Silz est évoqué, à propos de la chouannerie, par des auteurs comme Victor Hugo dans QuatrevingtTreize ou Paul Féval dans Chouans et Bleus ${ }^{2}$ mais ces romanciers ne disent rien de bien précis sur le Silz dont ils parlent ni sur sa famille.

La famille de La Haye de Silz est mal connue. Il n'existe pas d'archives familiales des La Haye de Silz et ce nom a disparu, le dernier à le porter

1. Les auteurs descendent d'Auguste de Silz, frère de Sébastien. La seconde fille d'Auguste de Silz, Clara de Silz, a épousé en 1817 Henry Le Masne, arrière-arrière-grandpère de Pierre Le Masne. Camille Le Masne est le fils de Pierre Le Masne.Les auteurs remercient les personnes sans lesquelles cet article n'aurait pu être écrit : Suzanne Cochois (également descendante de Clara de Silz) qui a reconstitué un historique de la famille de La Haye de Silz (publié par Roger Le Masne), en s'aidant de travaux de Yves Morineaux; Marcel Couëdel, originaire d'Arzal, qui nous a signalé les lettres de Sébastien de Silz à la Rossignolerie et les a transcrites; Olivier Hilbert (descendant d'Auguste de Silz par sa première fille Marie Cassienne) pour son travail généalogique; enfin Chantal Méxès pour son aide.

2. Féval, Paul, Chouans et bleus, Victor Palmé, 1879, p. 5-70. Hugo, Victor, QuatrevingtTreize, Quantin et $C^{\text {ie }}, 1874$; dans ce dernier roman, Hugo fait dire à La Vieuville : " À cette heure, dans cette armée de paysans, il y a des héros, il n'y a pas de capitaine. D’Elbée est nul. La Rochejaquelein est un magnifique sous-lieutenant; Silz est un officier de rase campagne impropre à la guerre d'expédients... " BALZAC, Honoré (de), s'intéresse dans Les Chouans, Le Livre de Poche, 1972 [1829], à une chouannerie plus tardive. Son roman se déroule à l'automne 1799, vers Fougères. 
étant décédé en $1903^{3}$. Des reconstitutions historiques d'origine familiale ${ }^{4}$ apportent toutefois des éléments. La famille de La Haye de Larré, de très ancienne noblesse bretonne, - on retrouve sa trace dès le XIv ${ }^{\mathrm{e}}$ siècle -, est installée de part et d'autre de la Vilaine. Elle possède le manoir de la Cour de La Haie à Larré ${ }^{5}$, le manoir de Coroller (aujourd'hui La Carolais, à Camoël), le château de Silz à Arzal. En 1673, Pierre de La Haye de Silz acheta le fief de Lauvergnac, fief qui joua un rôle dans le conflit de Sébastien de Silz avec son père.

Sébastien est le fils aîné des huit enfants que compte cette famille noble en 1791. Six s'engagent contre la Révolution. Un ou peut-être deux frères (Sébastien et Auguste, qui sont parfois confondus) font partie de la conspiration de La Rouërie. Trois frères (Sébastien, Auguste, Jacques) participent à l'insurrection du printemps $1793^{6}$; Jacques est arrêté et guillotiné en 1794, à 21 ans. Plusieurs sœurs sont également engagées ${ }^{7}$.

Sébastien de Silz a été incarcéré par lettre de cachet à Angers de 1781 à 1785, à la demande de son père et à la suite d'un conflit lié au décès de sa mère et au remariage de son père. Cet enfermement est évoqué par deux fonds d'archives : le fonds de l'intendance de Bretagne à Rennes retrace la procédure de son enfermement et de sa libération et un fonds des archives départementales du Morbihan donne des lettres de Sébastien lors de son emprisonnement. En s'appuyant notamment sur ces archives, cet article se propose deux objectifs liés : d'abord retracer la vie d'une famille intéressante du fait de son rôle en Bretagne durant la Révolution, et évoquer la vie de Sébastien de Silz et de sa famille avant la Révolution; ensuite évoquer les raisons de l'enfermement de Sébastien de Silz en 1781, et ses efforts pour sortir de prison.

Sébastien n'a pas eu une jeunesse exemplaire et le conflit avec son père ne peut s'expliquer seulement par la dureté de ce dernier : les familles de la noblesse sont, comme les autres, affectées par les décès et les remariages. Mais le conflit de Sébastien avec son père a abouti à un enfermement de quatre ans. Les circonstances de cette détention apportent divers éléments sur la société d'Ancien Régime. Cet enfermement montre, à partir d'un cas concret, le fonctionnement des lettres de cachet et les circuits administratifs de ce système. Le paiement de la pension par la famille est un préalable indispensable à tout enfermement. La vie de celui qui est enfermé n'est pas rose : les lettres de Sébastien montrent comment l'existence d'un jeune homme

3. Charles Pierre Marie Louis de La Haye de Silz, petit-fils de Jean-Marie de La Haye de Silz (1766-1799) est décédé à Vannes. Sébastien de Silz n'a pas eu d'enfant mais plusieurs de ses frères et sœurs en ont eu (voir tableau 1).

4. Cochols, Suzanne, La famille de La Haye de Silz, document manuscrit, reproduit en partie dans LE MASNE, Roger, Vingt générations de Gascons et de Bretons, 2 t., Viroflay, 1998.

5. Sur les murs de ce manoir du Xve siècle figurent encore les armes de la famille, " de gueules à trois étoiles d'or ".

6. Dupuy, Roger, De la Révolution à la Chouannerie, Flammarion, 1989 et Les Chouans, Hachette, 1997, donne certains éléments à leur propos.

7. Voir les notes du tableau 1 les concernant. 
plein de vie est bouleversée du jour au lendemain. Elles permettent de comprendre pourquoi l'enfermement dure quatre ans, alors que les faits de départ ne sont pas très graves. En outre, Sébastien engage, depuis sa prison, une procédure de retrait lignager; cette procédure d'Ancien Régime, plutôt désuète, aboutit à un résultat étonnant : le fils, enfermé par son père et sans ressource, finance la rente viagère de son père. Les hommes et les femmes que Sébastien sollicite pour sa libération dessinent des alliances entre une moyenne noblesse locale et des membres de la haute noblesse, qui ont un pied à Versailles. Au travers de l'enfermement de Sébastien, les relations de la famille de La Haye de Silz avec d'autres lignages de la même région, les Cramezel de Kerhué et les Le Sénéchal de Kerguizé, sont mises en lumière, apportant un éclairage original sur la moyenne noblesse du Vannetais.

Dans une première partie, nous décrivons la famille de Sébastien de La Haye de Silz et nous retraçons la rupture familiale consécutive au remariage de son père. Nous évoquons ensuite le conflit entre Sébastien et son père et l'enfermement qui en découle ( $2^{\mathrm{e}}$ partie), enfin la libération de Sébastien et sa vie jusqu'au décès de son père en 1791 ( $3^{\mathrm{e}}$ partie).

\section{La famille de La Haye de Silz et le décès de Marie Élie, mère de Sébastien}

Le père de Sébastien, Jean-Sébastien de La Haye de Silz, est né en 1727 à La Ville-au-Fevre (Saint-Nazaire). Il est comte de Larré, seigneur de Coscat (Arzal), du Plessis, de Lauvergnac, de Trescalan, de Clis, de Kermoisan (Guérande), de La Ville-au-Fevre (voir la carte 1). Il poursuit une carrière militaire, puis épouse en janvier 1750 à Missillac Marie Élie (parfois Eslye), âgée de 17 ans, également de très ancienne noblesse bretonne ${ }^{8}$. Les deux premiers enfants, des garçons nés à Saint-Nazaire et Pénestin, meurent en bas âge; puis vient Antoinette, née en 1753 à Saint-Nazaire. La famille s'installe en 1754 au château de Silz, que Jean-Sébastien reprend après le décès d'une cousine restée sans descendance. En ce château naît le 13 mars 1756 Sébastien Antoine Honoré Marie de La Haye de Larré de Silz, baptisé le lendemain en l'église d'Arzal; c'est l'aîné des garçons. Ensuite viennent d'autres enfants, jusqu'à Jacques en 1773. Au total 14 enfants ${ }^{9}$ (voir tableau 1), dont 10 atteignent l'âge adulte et 8 sont encore vivants au décès de leur père en 1791. La jeunesse de Sébastien, ainsi que celle de ses frères et sœurs, paraît heureuse tant que leur mère est vivante ${ }^{10}$.

8. Marie Élie est à l'occasion de ses fiançailles, en décembre 1749, décrétée de majorité par jugement du tribunal de Pénestin. Elle est assistée par son oncle René de Cramezel de Kerhué, époux de Madeleine Élie, sœur de sa mère.

9. NASSIET, Michel, Noblesse et pauvreté, la petite noblesse en Bretagne, Société d'histoire et d'archéologie de Bretagne, 1997, p. 259-260, montre qu'une très forte fécondité est caractéristique de la petite noblesse, mais peut concerner aussi la moyenne noblesse. Dans cette moyenne noblesse, certaines épouses mettent au monde plus de 20 enfants.

10. Selon Cochors, Suzanne, La famille de La Haye..., op. cit., "Jean-Sébastien est un personnage influent, riche, autoritaire, intéressé, Marie fut une épouse douce, une mère 
La famille, qui possède de nombreuses propriétés foncières, est à l'aise sur le plan économique. Elle ne fait cependant pas partie de la haute noblesse, au train de vie fastueux et qui accapare pensions et charges mais plutôt de la moyenne noblesse ${ }^{11}$, restée provinciale. Les de La Haye de Silz appartiennent à une noblesse d'épée, dans laquelle les garçons sont militaires, plutôt qu'à une noblesse de robe privilégiant des carrières administratives. La famille vit des revenus de ses terres, des soldes ou retraites militaires pouvant s'ajouter.

Un évènement imprévu survient à Arzal durant l'hiver 1759-1760 : plusieurs vaisseaux de ligne français, poursuivis par les Anglais jusque dans la Vilaine, s'abritent au port de Vieille-Roche ${ }^{12}$; ils y resteront bloqués plusieurs années. Des officiers sont parfois invités au château de Silz. JeanSébastien participe - c'est une source de revenus pour lui - à l'approvisionnement de ces vaisseaux bloqués sur la rivière, en bas de son château.

Marie Élie se remet difficilement de la naissance de Jacques au début de 1773; elle semble épuisée par des grossesses successives. Antoinette de Cramezel de Kerhué, sa cousine germaine (sa mère est également une Élie), née la même année que Marie (1732) mais restée célibataire, vient l'aider et s'installe au château de Silz ${ }^{13}$.

Marie Élie meurt le 19 décembre 1774 et est enterrée dans l'enfeu des Rhuys au couvent des jacobins de Guérande. Jean-Sébastien se remarie quelques mois plus tard, le 15 mai 1775 à Missillac. Il épouse, après dispense d'affinité, Antoinette de Cramezel de Kerhué; cette dernière est la sœur de Jacques de Cramezel (1742-1795) ${ }^{14}$, qui a accompagné Bougainville lors de son tour du monde sur La Boudeuse. Les enfants n'apprécient pas le remariage; aucun d'entre eux ne figure parmi les nombreux signataires de l'acte de mariage.

Jean-Sébastien et sa nouvelle épouse partagent leur temps entre le château de Silz, l'été, et, l'hiver, un hôtel que Jean-Sébastien a loué à Vannes, après la mort de sa première épouse, pour 500 livres par an; cet hôtel est situé rue des Cordeliers. Jean-Sébastien dispose d'une chaise à porteurs pour son épouse, de deux voitures et de trois chevaux ${ }^{15}$.

attentionnée, pleine de tendresse et d'affection pour ses enfants. "

11. MEYER, Jean, La noblesse bretonne au XVIII siècle, Paris, SEVPEN, 1966, p. 24, donne de nombreuses indications sur cette moyenne noblesse qui représente $30 \%$ du total de la noblesse dans le Vannetais, plus que dans d'autres parties de la Bretagne.

12. PluyetTe, Henri, Le blocus de la Vilaine 1759-1762, édité par l'auteur, 1980. L'un de ces vaisseaux est Le Glorieux, sur lequel sert ensuite Auguste de Silz et sur lequel il est blessé au combat des Saintes en 1782. Dans sa correspondance de 1760 avec son frère le marquis de Mirabeau, le bailli de Mirabeau, officier en tournée d'inspection, évoque ces bateaux coincés dans la Vilaine (Musée Paul Arbaud, Aix-en-Provence, Dossier 25). Il est reçu en Bretagne chez la duchesse de Lorge, que Sébastien de Silz tentera d'impliquer dans sa libération.

13. Cochors, Suzanne, La famille de La Haye..., op. cit.

14. Il sera fusillé en 1795 à Vannes, pour avoir pris part à l'expédition royaliste de Quiberon.

15. Ces renseignements très précis proviennent du rapport administratif de Thomas de Bénéac, subdélégué à La Roche-Bernard (Arch. dép. d'Ille-et-Vilaine, C 161, dossier 


\section{Figure 1 - Principaux lieux cités en Bretagne}

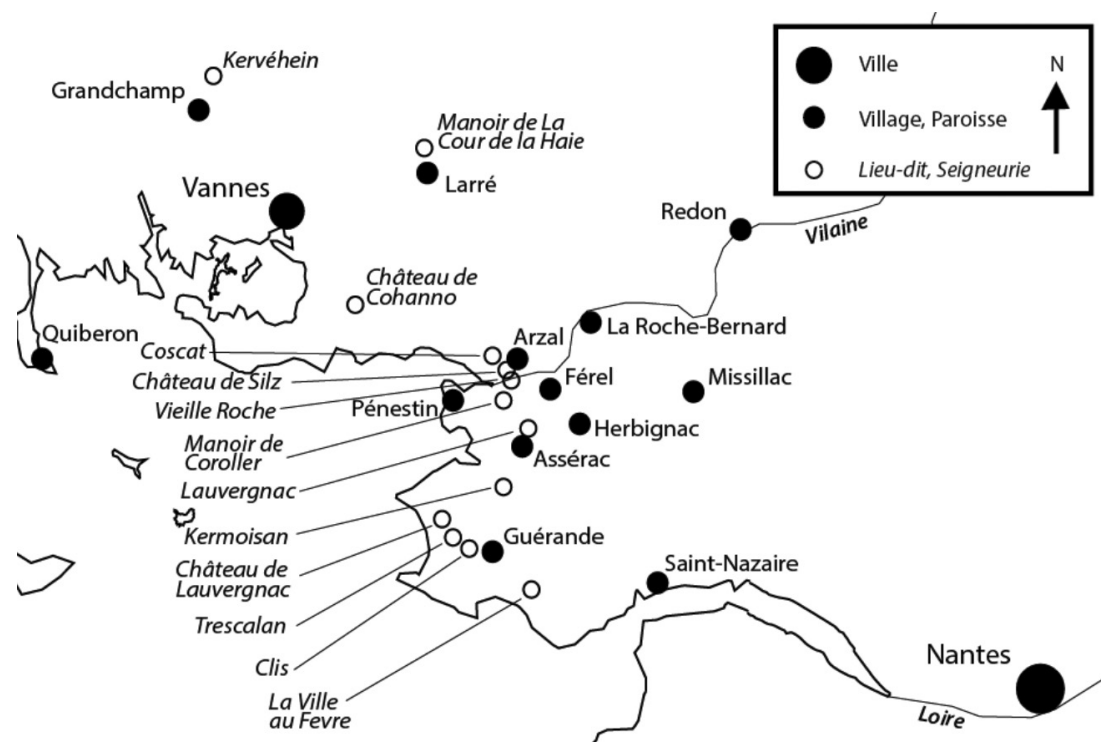

D'après Thomas de Bénéac (subdélégué de l'intendant de Bretagne à La Roche-Bernard), trois des quatre sœurs de Silz sont en pension dans des couvents, chacune pour 200 livres par an ${ }^{16}$ : l'aînée, Antoinette, dans un couvent près de Rennes, deux autres chez les carmélites de Ploërmel ${ }^{17}$;

Sébastien de La Haye de Silz, lettre du 6 avril 1783 à l'intendant de Bretagne). À propos des subdélégués de l'intendance de Bretagne, FrévILLE, Henri, L'intendance de Bretagne, Rennes, Plihon, 1953, 3 t., ainsi que DiDIER, Sébastien, « Du réseau au maillage administratif, la construction territoriale des subdélégations bretonnes de 1689 à 1789 ", Annales de Bretagne et des Pays de l'Ouest, 2014, nº 4, p. 81-106.

16. Il n'est pas possible de donner un équivalent monétaire en euros de la livre tournois, mais les salaires de l'époque permettent des comparaisons. Selon MorRISSON, Christian et SNYDER, Wayne, "Les inégalités de revenus en France du début du XVIII ${ }^{\mathrm{e}}$ siècle à 1985 ", Revue économique, p. 119-154, janvier 2000, le revenu annuel d'un salarié agricole en 1788 est en moyenne de 160 livres. Les différences entre régions sont importantes.

17. Il ne s'agit pas ici d'un enfermement du type de celui décrit par Diderot dans La Religieuse. Certaines filles de la noblesse étaient placées dans des couvents, au-delà de l'âge des études, et des veuves pouvaient également s'y réfugier. Louis XV avait placé plusieurs de ses filles pendant 10 ans à l'abbaye de Fontevrault. CHODERLOS DE LACLOS, Pierre, Les Liaisons dangereuses, Euvres complètes, NRF-La Pléiade, 1979 [1782], Lettre Première, p. 11-12, évoque les bonnes conditions de vie de Cécile Volanges dans une pension religieuse. Certains couvents faisaient payer des pensions importantes, jusqu'à plusieurs milliers de livres par an, aux familles de ces jeunes pensionnaires. Les conditions de vie pouvaient être agréables, voire luxueuses. Mais le montant des pensions versées par Jean-Sébastien pour ses filles est réduit, 200 livres par an pour chacune; les conditions de vie étaient sans doute spartiates chez les carmélites de Ploërmel, et le degré de liberté réduit. Sébastien de Silz affirme que ses sœurs ont été placées dans des couvents par son père, à la demande de sa belle-mère. 


\section{Tableau 1 - Les enfants de Jean-Sébastien de Silz (1727-1791) et de Marie Élie (1732-1773)}

\begin{tabular}{|c|c|}
\hline 1 & $\begin{array}{l}\text { Jean-Marie, baptisé le } 21 \text { décembre/1750 à Saint-Nazaire, décédé } 13 \text { janvier/1751 à } \\
\text { Pénestin. }\end{array}$ \\
\hline 2 & Jean-Marie, baptisé le 29 mars/1752 à Pénestin, décédé en 1752. \\
\hline 3 & $\begin{array}{l}\text { Antoinette Renée Marie, née le } 2 \ldots \text { septembre } 1753 \text { à Saint-Nazaire, épouse le } 11 \text { floréal } \\
\text { An II ( } 30 \text { août 1794) Samuel Robin, officier républicain; décédée le } 12 \text { octobre } 1820 \text { à } \\
\text { Nantes [ } 1 \text { garçon]. } \\
\text { [Le deuxième chiffre de son jour de naissance est illisible sur l'acte; elle est née entre le } 20 \\
\text { et le } 29 \text { septembre } 1753 \text {. Antoinette de Silz est arrêtée en avril } 1793 \text { puis condamnée à mort } \\
\text { en } 1794 \text { pour activités contre-révolutionnaires; elle échappe à la guillotine en acceptant la } \\
\text { proposition de mariage d'un officier républicain. Sur ce point, CHASSIN, Charles Louis, Les } \\
\text { pacifications de l'Ouest } 1794-1801 \text {, Paris, Dupont, } 3 \text { t., 1897, t. II, p. 553; Gabory, Émile, Les } \\
\text { Vendéennes, Paris, Perrin, 1935, p. 189.] }\end{array}$ \\
\hline 4 & $\begin{array}{l}\text { Sébastien Antoine Honoré Marie, né le } 13 \text { mars } 1756 \text { à Arzal, baptisé le lendemain, } \\
\text { décédé le } 9 \text { prairial an III ( } 28 \text { mai 1795) à Grandchamp }\end{array}$ \\
\hline 5 & $\begin{array}{l}\text { Marie-Françoise, née le } 18 \text { juin } 1757 \text { à Arzal, épouse René Osmond de Centeville (dates } \\
\text { de mariage et de décès inconnues) [1 garçon]. } \\
\text { [Marie-Françoise est arrêtée en mars } 1793 \text { puis relâchée. Elle s'oppose en } 1796 \text { à l'arresta- } \\
\text { tion de son frère Auguste.] }\end{array}$ \\
\hline 6 & $\begin{array}{l}\text { Auguste Marie, né le } 9 \text { novembre } 1760 \text { à Arzal, épouse le } 10 \text { janvier } 1792 \text { à Arzal Jeanne } \\
\text { Marie de Kermasson de Kervail (1773-1807), décédé le } 4 \text { janvier } 1822 \text { à Arzal [2 filles]. } \\
\text { [Auguste est un des chefs chouans du Morbihan. Il se rend en } 1796 \text { aux autorités républi- } \\
\text { caines, mais, contrairement aux accords de reddition, il est condamné à la déportation en } \\
\text { Guyane et enfermé } 3 \text { ans à la prison de Saint-Martin-de-Ré.] }\end{array}$ \\
\hline 7 & Anne Marie Louise, née le 25 janvier 1762 à Arzal, décédée le 4 octobre/1789 à Guérande. \\
\hline 8 & Armand Désiré Marie, né le 12 octobre 1763 à Arzal, décédé le 21 février/1765 à Arzal. \\
\hline 9 & Anne Louise Marie, née le 16 octobre 1764 à Arzal, décédée en bas-âge à une date inconnue. \\
\hline 10 & $\begin{array}{l}\text { Jean-Marie, né le } 20 \text { février } 1766 \text { à Arzal, épouse Jeanne Philippe (1762-1827) le } 7 \text { février } \\
1792 \text { à Pénestin, décédé le } 2 \text { germinal an VII ( } 22 \text { mars/1799) à Pénestin [ } 2 \text { garçons, dont } 1 \\
\text { décédé en bas-âge]. }\end{array}$ \\
\hline 11 & Cassien Jean-Marie, né le 3 mars 1767 à Arzal, prêtre, décédé avant son père (date inconnue). \\
\hline 12 & $\begin{array}{l}\text { Louis Marie, né le } 29 \text { juillet } 1768 \text { à Arzal, épouse le } 21 \text { nivôse an I (10 janvier 1793) Marie } \\
\text { Hardoin à Saint-Nazaire, puis (après le décès de celle-ci) le } 30 \text { thermidor an VII (17 août } \\
\text { 1799) à Herbignac Marie-Jeanne Lizeul (1772-1814), décédé le } 1^{\text {er }} \text { Thermidor an IX } \\
\text { (20 juillet 1801) à Saint-Nazaire [pas de descendance connue]. }\end{array}$ \\
\hline 13 & $\begin{array}{l}\text { Eugénie Armande, née le } 6 \text { février } 1771 \text { à Arzal, épouse le } 13 \text { août } 1792 \text { à Arzal Jean Ben- } \\
\text { jamin Thomas de La Borde [1 garçon et } 1 \text { fille], date de décès inconnue. } \\
\text { [Benjamin Thomas de La Borde est révoqué de son poste d'officier judiciaire à La Roche- } \\
\text { Bernard pour sympathies royalistes.] }\end{array}$ \\
\hline 14 & $\begin{array}{l}\text { Jacques Marie, né et ondoyé le } 10 \text { février 1773, baptisé le } 2 \text { juillet 1788, condamné à mort } \\
\text { et guillotiné à Nantes, le } 28 \text { ventôse an II (18 mars 1794). } \\
\text { [Jacques de Silz a participé à l'insurrection du Morbihan de 1793. Il est arrêté, condamné } \\
\text { à mort et guillotiné place du Bouffay à Nantes. Sur ce point, Arch. dép. de Loire-Atlantique, } \\
\text { L 1502, et LaLLlé, Alfred, La Justice révolutionnaire à Nantes et en Loire-Inférieure, Les } \\
\text { Éditions du Choletais, 1991, p. 132. Les descendants de Jacques de Silz ont reçu en } \\
1828 \text { une indemnité concernant son exécution. (État détaillé des liquidations faites par la } \\
\text { Commission d'indemnités du } 31 \text { décembre 1827, Paris, Imprimerie nationale, 1828, vol. 2, } \\
\text { p. 437-438). Tous les frères et sœurs de Jacques de Silz sont décédés à cette époque. } \\
\text { Reçoivent des indemnités : Pierre Marie de La Haye de Silz (fils de Jean-Marie), Marie- } \\
\text { Cassienne Piard-Deshayes (fille d'Auguste), Clara Marie Le Masne (fille d'Auguste), } \\
\text { Prudent Thomas de La Borde (fils d'Eugénie Armande), Jean-Pierre Osmond (fils de Marie- } \\
\text { Françoise), enfin Armand Robin (fils d'Antoinette). Le montant total des indemnités est } \\
\text { réduit, } 250 \text { francs au total. Armand Robin reçoit moitié moins que les autres descendants, } \\
\text { compte tenu des circonstances très particulières du mariage de ses parents.] }\end{array}$ \\
\hline
\end{tabular}


la dernière sœur habite avec son père et sa belle-mère. Le plus âgé des 5 frères de Sébastien, Auguste, est officier de marine; les quatre autres sont au collège à Vannes.

Thomas de Bénéac note en 1783 que M. de La Haye de Silz lui parait " peu heureux en enfants ". Quelques années auparavant, une de ses filles a quitté nuitamment le domicile familial avec un garçon cuisinier et s'est placée comme domestique chez un cafetier. Son père l'y a fait arrêter et conduire chez les carmélites de Ploërmel, où elle est encore. Le père de Sébastien a envoyé en 1776 son fils à Rennes pour qu'il apprenne le métier des armes, payant à cet effet une pension de 400 livres par an mais Sébastien a entretenu à Rennes de très mauvaises fréquentations et s'est mis à boire. Il s'est engagé dans un régiment de dragons ${ }^{18}$. Sa conduite y fut si mauvaise - du moins c'est ce qu'affirme Thomas de Bénéac - que des officiers du régiment écrivirent à son père pour lui demander de l'en retirer. Au printemps 1781, Sébastien, qui n'est plus dans son régiment, est employé surnuméraire dans les Fermes des devoirs de Bretagne ${ }^{19}$. Il a donc cherché une réorientation : les emplois dans les fermes des impôts indirects offrent de nombreux emplois, très prisés, et qui constituent une alternative à une carrière militaire ${ }^{20}$.

Les lettres de Sébastien concordent sur de nombreux points avec les informations données par Thomas de Bénéac mais insistent sur le peu d'intérêt apporté par le père et la belle-mère aux enfants. Sébastien reproche à son père d'enfermer ses sœurs dans des couvents et de placer ses frères, très jeunes, dans les gardes de la marine à Brest (lettre du 17 décembre 1783) ${ }^{21}$. Le reproche de Sébastien paraît fondé, car ses sœurs sont au couvent et ne se marient pas : en 1783, Antoinette a 30 ans, et MarieFrançoise 26 ans. Aucun mariage d'enfant n'interviendra avant le décès de Jean-Sébastien en 1791; quatre enfants se marient alors très vite, en 1792 et 1793. Jacques avait été seulement ondoyé à sa naissance en 1773 et l'état de santé de sa mère n'avait pas permis qu'il soit baptisé de son vivant; il

18. Sa carrière militaire n'a pu être reconstituée. Nous avons consulté aux Archives militaires de Vincennes les livres de nombreux régiments de dragons, parmi les 24 qui existent en 1781, sans retrouver trace de Sébastien de Silz.

19. La Ferme des devoirs est l'organisme chargé de la perception des impôts et droits sur les boissons en Bretagne.

20. NASSIET, Michel, Noblesse..., op. cit., p. 349-351 indique que 189 gentilshommes étaient employés dans les fermes indirectes en Bretagne en 1729, et que 64 surnuméraires attendaient un emploi. Selon AzIMI, Vida, "Le traitement des agents publics sous l'ancien régime ", Revue historique de droit français et étranger, 1989, nº 3, p. 429-468, les surnuméraires n'étaient pas rémunérés en attendant d'obtenir un poste et restaient à la charge des familles.

21. Auguste de Silz commence sa carrière comme garde-marine à 18 ans (Arch. nat., F/7/1760, dossier d'Auguste de La Haye de Silz). Jean-Marie et Louis Marie ont été également gardes-marine, après le collège. NASSIET, Michel, Noblesse..., op. cit., p. 355-356, précise que seule la moyenne noblesse pouvait envisager de placer ses enfants dans les garde-marine. Il fallait des recommandations importantes pour y entrer et payer une pension d'environ 600 livres par an, qui n'était pas à la portée de la petite noblesse. 
ne fut baptisé qu'en 1788, à l'âge de 15 ans $^{22}$ : la question de son baptême a été oubliée après le décès de sa mère.

Les tensions entre les enfants de Silz et Antoinette de Cramezel et sa famille persistent jusqu'en 1792-1793 : aucun Cramezel de Kerhué n'est invité à signer les actes de mariage d'Auguste (1792), Jean-Marie (1792), Eugénie Armande (1792), ou Louis Marie de Silz (1793).

\section{La personnalité de Sébastien de La Haye de Silz}

Sébastien est allé au collège, sans doute à Vannes; ses lettres indiquent une bonne connaissance de la langue française et une certaine culture. On dispose à son propos d'une description de police, ainsi que pour son frère Auguste, donnée après l'insurrection du printemps 1793; elle n'est pas bienveillante :

"Signalement des deux de Silz qui ont été à la tête des brigands. L'aîné des deux, âgé d'environ 39 à 40 ans, né à Silz paroisse d'Arzal, district de La Roche-Sauveur, taille environ 5 pieds 3,4 pouces, fluette, bien étagée, droit, beaucoup gravé de petite vérole, le teint bourgeonné comme un yvrogne, physionomie ronde et intéressante, il mâche beaucoup de tabac surtout en poudre. Il a servi quelque temps dans un régiment de dragons, il en est sorti avec une cartouche jaune. C'est lui qui au mois de mars dernier a livré aux brigands la ville de Guérande ${ }^{23} \ldots$ ".

22. Le parrain est Jacques de Cramezel, et la marraine une de ses sœurs. Ils avaient été choisis du temps de Marie Élie, comme l'indique le prénom de Jacques, qui est celui de son parrain.

23. Arch. dép. du Morbihan, L 1529 et Nicol, Pierre, "Un soulèvement en frimaire an II ", Revue morbihannaise, 1903, n ${ }^{\circ} 11$, p. 353-368, et $\mathrm{n}^{\circ} 12$, p. 46-59. La description ajoute : "Il était commandant de la Garde Nationale : il est marié à Guérande. Depuis il a erré dans le département du Morbihan; il a été caché longtemps chez son frère, le chevalier, à Vieille-Roche, paroisse d'Arzal ". Sébastien n'était pas marié, contrairement à ce qu'affirme la description précédente, mais aimait certainement les femmes. CADIC, François, Histoire populaire de la Chouannerie en Bretagne, Rennes, Presses universitaires de Rennes, 2003, t. 1, p. 548-549, note qu'en 1795 à Grandchamp, " Le comte de Silz, tué en combattant, avait passé la revue des soldats en compagnie de sa maîtresse à cheval derrière lui ». La description donnée d'Auguste de Silz est la suivante : "Chevalier, âgé d'environ 32 ans, est né à Silz, paroisse d'Arzal; taille environ 5 pieds 1 pouce, gros, trapu, bien constitué, la jambe forte, un peu arquée, le visage gros, rond, un peu soufflé; bonne carnation; les yeux un peu enfoncés et petits, bouche petite; les lèvres épaisses, un peu élevé surtout la supérieure. Il porte ordinairement un chapeau à cuve élevée; il a servi dans la marine ci-devant royale; il était lieutenant de vaisseau quand il a été chassé. Il est marié à Vieille-Roche avec une demoiselle Kermasson Kerval qui est au Petit Couvent. C'est lui qui était à la tête des brigands de Rochefort. Tout l'été dernier, il travaillait avec son frère aîné dans la paroisse de Berric, Sulniac, Lauzach, comme journalier. Il a été caché quelque temps chez son fermier à Coscat, distance de Vieille-Roche d'environ une portée de fusil à balle. " Le Petit Couvent est la prison de Vannes où l'épouse d'Auguste a été internée en 1793 avec sa fille de 3 mois et son père. Auguste et son épouse demeurent dans leur maison de Vieille-Roche (appelée aujourd'hui "Caserne des douaniers"). 
Cette « cartouche jaune " signifie que les déboires militaires de Sébastien étaient connus ${ }^{24}$.

Un témoignage plus favorable est celui du Vendéen Bertrand Poirier de Beauvais ${ }^{25}$, qui rencontre Sébastien de Silz en 1795 au moment de la signature des accords de La Mabilais (à Rennes), et se lie d'amitié avec lui. Poirier de Beauvais, souvent critique vis-à-vis des chefs chouans, parle à propos de Sébastien de sa loyauté, de sa bravoure, de son désintéressement, le voit comme un être sensible, d'" une délicatesse qu'il est difficile de peindre, d'un cour aimant, fait pour n'avoir que des amis en ce monde ».

Sébastien est installé en 1781 à Guérande, où il mène joyeuse vie. Peu avant sa majorité - il a 25 ans en mars 1781 -, son père, mécontent de son comportement et peut-être inquiet de l'approche de cette majorité, rédige un placet demandant son enfermement par lettre de cachet du roi.

\section{Lettre de cachet et arrestation de Sébastien}

L'arrestation de Sébastien résulte d'une procédure compliquée. La délivrance de lettres de cachet manifeste la justice personnelle du roi. Certaines lettres de cachet correspondent à la volonté du souverain d'enfermer des opposants mais, le plus souvent, les lettres de cachet sont rédigées par l'administration royale à la demande des familles : le roi entend les protéger notamment contre la prodigalité ou les mésalliances. Des affaires sensibles, concernant souvent des familles nobles ou des ecclésiastiques, sont ainsi traitées discrètement ${ }^{26}$. La procédure est la suivante, selon J. Hautebert ${ }^{27}$ : la famille écrit un placet à l'intendant de la province; ce placet détaille les faits répréhensibles qui motivent la demande d'enfermement. Le mémoire est signé par le principal requérant auxquels s'associent d'autres demandeurs, qui peuvent être de la famille ou des extérieurs ${ }^{28}$. Le placet précise le lieu d'enfermement, ce qui suppose l'accord préalable du responsable de ce lieu, et l'accord de la famille pour payer la pension; le lieu d'enfermement

24. Selon NICOL, "Un soulèvement... ", art. cit., on donnait le nom de cartouche sous l'Ancien Régime à la feuille remise au soldat à son départ de l'armée. Elle était blanche, verte, rouge ou jaune selon que l'homme était simplement libéré, réformé, puni ou dégradé. Selon CORvISIER, André, Histoire militaire de la France, Paris, PUF, 1992, t. 2, p. 46, une cartouche jaune était crainte des soldats; en effet, s'ils voulaient se réengager, ils avaient intérêt à cacher leur passé militaire et à recommencer leur carrière militaire sans aucune ancienneté.

25. PoIRIER De Beauvals, Bertrand, Mémoires inédits, Paris, Plon, 1893, p. 372.

26. À propos des lettres de cachet, QueTEL, Claude, De par le Roy, Toulouse, Privat, 1981, ainsi que RoLLAND, Muriel, "L'exercice de la puissance paternelle sur les mineurs en Bretagne (1731-1789) ou les moyens d'éviter les mésalliances ", dans Plessix-BuISSET, Christiane (dir.), Ordre et désordre dans les familles, Rennes, PUR, 2002, p. 12-49.

27. HAUTEBERT, Joël, L'internement par lettre de cachet d'après les archives de l'intendance de Bretagne 1769-71 et 1786-89, Mémoire de DEA, Université de Rennes, 1990.

28. Le livre de FARGE, Arlette et Foucault, Michel, Le désordre des familles (lettres de cachet des archives de la Bastille), Paris, Gallimard, 1982, donne certains dossiers de ce type. 
peut être extérieur à la province, sans pour autant que soit dessaisi l'intendant qui a reçu le placet. Ce dernier est ensuite envoyé au secrétaire d'État à la maison du Roi qui, s'il est d'accord, réexpédie le placet à l'intendant et lui demande de s'enquérir de la véracité des faits énoncés. L'intendant fait faire une enquête sur place par son subdélégué local puis écrit au secrétaire d'État à la maison du Roi et émet un avis. Si la maison du Roi décide d'octroyer les " ordres du Roi ", la lettre de cachet est expédiée à l'intendant en vue de son exécution. Lors de l'arrestation, l'officier de police présente la lettre de cachet à la personne arrêtée, qui est transférée dans le lieu de détention prévu. Le responsable du lieu d'enfermement signe la lettre de cachet, qui ne précise pas le motif d'enfermement.

Les demandes de lettre de cachet sont nombreuses au XVIII ${ }^{\mathrm{e}}$ siècle, en Bretagne comme ailleurs. Les fonds de l'intendance de Bretagne aux archives départementales d'Ille-et-Vilaine comportent 1700 dossiers de demandes de lettre de cachet pour les années 1731-1789; elles proviennent de familles à $78 \%{ }^{29}$. L'un des lieux d'enfermement, mentionné par J. Hautebert ${ }^{30}$ et utilisé par les familles bretonnes, bien que situé en Anjou, est la maison de force des frères des Écoles chrétiennes d'Angers, La Rossignolerie ${ }^{31}$.

\section{Première demande d'enfermement de Jean-Sébastien, au début de 1781}

Le dossier de Sébastien de Silz à l'intendance de Bretagne comprend 17 pièces, relatives à l'enfermement de 1781, à la mise en cause de cet enfermement en 1783, enfin à la libération de 1785. Il est néanmoins incomplet et ne contient pas les deux placets envoyés en 1781 par Jean-Sébastien pour demander l'enfermement de son fils, ni celui de Sébastien en 1783 pour demander sa libération.

La première demande de Jean-Sébastien est de janvier 1781. Elle ne figure pas dans le dossier, et on ne sait pas si elle a été faite auprès de l'intendance de Bretagne, ou directement auprès de M. Amelot ${ }^{32}$, secrétaire d'État à la maison du Roi à Versailles. Elle est en tout cas répercutée auprès de l'intendance de Bretagne : Jean-Sébastien demande à ce que son fils soit enfermé à la prison du Taureau ${ }^{33}$ mais il ignore que cette prison n'accueille plus de pensionnaire à cette période; il ne s'est donc pas entendu préala-

29. Rolland, Muriel, " L'exercice... ", art. cit., p. 15.

30. HAUTEBERT, Joël, L'internement..., op. cit., p. 53.

31. Meyer, Jean, La Noblesse..., op. cit., t. 2, p. 1085-1087, montre que le recours aux lettres de cachet a fortement augmenté au sein de la noblesse bretonne au cours du $\mathrm{XVIII}{ }^{\mathrm{e}}$ siècle, et donne une liste des lieux de détention.

32. Antoine Jean Amelot de Chaillou (1732-1795) est secrétaire d'État de la maison du Roi de mai 1776 à novembre 1783. Il est chargé à ce titre des lettres de cachet.

33. Le château du Taureau, fort militaire situé en mer au nord de la baie de Morlaix, sert aussi de prison. Il a accueilli à certaines périodes des gentilshommes enfermés par lettre de cachet, comme le procureur La Chalotais en 1765. 
blement avec le responsable d'un lieu d'enfermement et sa demande ne peut alors aboutir. La demande de janvier 1781 est envoyée par l'intendant de Bretagne à son subdélégué à La Roche-Bernard, Thomas de Bénéac. Ce dernier explique par courrier à Jean-Sébastien de Silz qu'il doit trouver luimême un lieu de détention. Jean-Sébastien de Silz vient le voir à La RocheBernard, puis lui écrit le 17 mars qu'il a contacté le supérieur de l'hospice de Saint-Méen à Rennes; ce dernier n'a pas de place $^{34}$.

Jean-Sébastien écrit le 9 avril 1781, directement à Versailles, semble-til, pour dire qu'il est peu fortuné, que le prix de la pension de Saint-Méen lui convient, mais que, comme il n'y a pas de place pour le moment, son fils pourrait être provisoirement enfermé dans une prison de Rennes. M. Amelot écrit le 12 avril à l'intendant de Bretagne, Caze de la Bove, en lui renvoyant cette lettre; l'intendant transmet à son subdélégué le 18 avril et lui demande son avis. Thomas de Bénéac répond le 24 avril; il confirme le contenu du placet de Jean-Sébastien, de façon sévère pour Sébastien :

" Dans le temps mesuré où je reçus vos ordres, ce fils scandaleux et vraiment méprisable étoit depuis plusieurs jours dans un cabaret de notre ville avec un jeune homme de sa trempe, et lui et d'autres y donnaient des preuves les plus éclatantes d'inconduite; d'ailleurs depuis longtemps, je suis personnellement convaincu de la crapule inconcevable dans laquelle croupit depuis plusieurs années M. de Silz fils ".

Selon Thomas de Bénéac, Jean-Sébastien hésite sur l'enfermement de son fils, notamment à cause du prix de la pension. Jean-Sébastien écrit de son côté à l'intendant de Bretagne le 25 avril, explique qu'il ne trouve pas de place et lui demande de trouver une place, pour un maximum de 400 livres par an. L'intendance de Bretagne considère que ce n'est pas son rôle et lui renvoie son placet fin avril.

\section{La majorité de Sébastien et ses conséquences}

Sébastien devient majeur le 13 mars 1781. Les biens de sa mère Marie Élie font l'objet d'un acte de partage le 16 mai $1781^{35}$. Il est le seul enfant concerné; Antoinette, également majeure, ne bénéficie pas du partage, ne l'ayant peut-être pas exigé. Il hérite de sa mère, et donc de la famille Élie, d'une maison au bourg de Missillac, et de terres affermées. Les fermages rapportent 189 livres par an. La propriété sert de base au versement d'une rente établie sur la base d'une valeur de la propriété de 1120 livres. L'héritage de Sébastien est soumis à de fortes contraintes, sans doute voulues par son père et il ne peut en espérer qu'une cinquantaine de livres de revenu par an. Un certain Chailloux de l'Étang perçoit les revenus de la propriété et verse une rente à partir de ces revenus, pour $2 / 3$ aux demoiselles Michelle et Jeanne Françoise Cramezel de Kerhué (sœurs d'Antoinette de

34. Arch. dép. d'Ille-et-Vilaine, C 161. Lettre du 24 avril de Thomas de Bénéac à l'intendant de Bretagne.

35. Arch. dép. de Loire-Atlantique, E 1344. 
Cramezel) et pour 1/3 à Marie Élie (ou son époux) ${ }^{36}$. Sébastien de Silz s'engage à en rester au système en cours, ou, s'il en change, à payer lui-même la rente des demoiselles de Kerhué. Jean-Sébastien a gardé le droit de stocker ses récoltes dans un grenier de la maison de Missillac.

Sébastien dépense trop; en particulier, il achète de beaux vêtements ${ }^{37}$. Pour pouvoir régler ses achats, il vend à Jean Loiseau, marchand de bois, le 21 juin 1781, des terres de Missillac pour environ 400 livres, et le 22 août 1782 la maison de Missillac et d'autres terres pour 1500 livres. Dans les deux cas il est prévu qu'une partie de la somme sera payée par Loiseau après la vente. Le 22 août, apparaît dans les actes un certain Le Pied, marchand de draps de soie à Guérande, à qui Sébastien doit de l'argent. Le Pied accepte d'être payé par ce dernier en échange d'une créance d'environ 200 livres qu'il a sur Loiseau depuis le 21 juin, correspondant à la partie non payée de la première vente.

Loiseau ne verse pas tout qu'il doit à Sébastien de Silz; Le Pied n'est pas non plus payé par Loiseau et se retourne contre ce dernier. Les demoiselles de Cramezel, inquiètes pour le paiement de leur rente, intentent dès juillet 1781 une action contre Loiseau au tribunal de La Roche-Bernard, en demandant que les biens achetés par Loiseau à Missillac soient saisis à titre conservatoire ${ }^{38}$. Elles mettent en garde Sébastien de Silz, dont elles affirment que les dépenses sont considérables; elles lui rappellent qu'il est responsable du paiement de leur rente. Elles intentent ensuite plusieurs autres actions au tribunal, notamment une action en retrait lignager ${ }^{39}$ pour reprendre à Loiseau les biens de Missillac; en mars 1782, elles assignent Sébastien de Silz et lui demandent de payer 1200 livres. Entre-temps, fin janvier 1782, Jean-Sébastien de Silz est intervenu au même tribunal de La Roche-Bernard : il expose que son fils est maintenant enfermé en vertu d'ordres du roi dans la maison de la Rossignolerie à Angers et ne peut plus gérer ses biens. Il a obtenu du présidial de Vannes le 21 janvier 1782 le droit de les administrer à sa place ${ }^{40}$. Il fait sommation à Loiseau de payer et

36. Le système a été mis en place longtemps avant, sur la base de biens de la famille Élie, avec l'accord de Marie Élie.

37. Après son arrestation à Guérande, son logeur, $\mathrm{M}^{\mathrm{e}}$ Hardoin envoie à Guibert un état des effets que Sébastien vient d'acheter et qui sont restés chez lui : " un petit chapeau bordé en or, un fraque bord galonné en argent, une veste bleue, une culotte de prunelle, un gillet de cotton croisé, quatre chemises, huit cols, 2 mouchoirs, deux paires de bas de laine et deux de fil ". Dans une lettre (12 octobre 1783), Sébastien dit que ces achats ont coûté 25 louis (75 livres).

38. Une lettre de Sébastien de Silz (23 novembre 1783) parle de Loiseau et des demoiselles de Cramezel.

39. Nous expliquons un peu plus loin ce qu'est cette procédure. L'ensemble des documents relatifs à cette affaire figure aux Arch. dép. de Loire-Atlantique, E 1344, Famille de Cramezel de Kerhué. La procédure continue jusqu'en 1784. Les sœurs de Cramezel l'emportent sur Jean-Sébastien de Silz en ce qui concerne le retrait lignager : il s'agit d'un bien de la famille Élie. Jean-Sébastien de Silz leur transmet en 1784 les titres de propriété qui n'ont pas été donnés en 1781 à Sébastien.

40. Sur ce point, Arch. dép. du Morbihan, B 749, Présidial de Vannes. 
s'engage pour lui-même dans une procédure de retrait lignager, concurrente de celle des demoiselles de Cramezel $^{41}$.

Sébastien a agi de façon irréfléchie en vendant la propriété de Missillac; les sommes en jeu ne sont pas très importantes, mais il s'est attiré de nombreuses inimitiés, notamment au sein de sa famille.

\section{Aboutissement de la lettre de cachet}

Les évènements précédents ont incité Jean-Sébastien à reprendre la recherche d'un lieu d'enfermement pour son fils. Il écrit début septembre à Versailles pour dire qu'il est tombé d'accord avec la maison des frères des Écoles chrétiennes d'Angers. M. Amelot écrit à l'intendant de Bretagne à ce propos le 9 septembre $1781^{42}$ et répond parallèlement à Jean-Sébastien qu'il n'a pas de dossier au nom de Sébastien : il n'y a plus de procédure en cours, puisque la procédure précédente a été interrompue.

Jean-Sébastien s'adresse le 10 novembre 1781 à l'intendant de Bretagne, se plaint du rejet de sa demande précédente et renvoie un nouveau dossier complet, avec l'accord du Supérieur de la maison d'Angers, le frère Liboire. L'intendant de Bretagne envoie ce dossier le 20 novembre à M. Amelot; il donne son accord pour une lettre de cachet et justifie en même temps l'arrêt de la procédure précédente :

« Je crois donc que pour arrêter les désordres du sieur de La Haye, il est à propos d'accorder à son père les ordres du Roi qu'il sollicite. Si j'ai tant tardé à vous rappeler cette affaire, c'est que le père du sieur De La Haye ne pouvant trouver de maison dont le prix put lui convenir, et d'ailleurs espérant toujours que son fils reviendrait de ses égarements, on lui avait renvoyé toutes ses pièces le 25 avril dernier."

Le dossier comprend une pièce par laquelle Jean-Sébastien s'engage à payer 522 livres. Le 23 novembre, M. Amelot donne son accord et joint la lettre de cachet. Le secrétaire de l'intendant de Bretagne, Claude Petiet, écrit le 28 novembre à Thomas de Bénéac, lui donne l'ordre d'arrêter Sébastien et joint la lettre de cachet. Thomas de Bénéac répond le 8 décembre 1781 : il vient d'envoyer une brigade de la maréchaussée de La Roche-Bernard pour arrêter Sébastien et le conduire à Angers. La brigade a reçu une somme de 522 livres pour une année de pension ainsi que pour les frais de capture et de transport de Sébastien ${ }^{43}$. Ce 8 décembre 1781, Sébastien de Silz est arrêté à Guérande, dans le logement qu'il loue chez $\mathrm{M}^{\mathrm{e}}$ Hardoin; il est

41. Ce retrait lignager permettrait à Jean-Sébastien ou aux demoiselles de Cramezel d'annuler la vente à Loiseau, en se substituant à Sébastien de Silz. Loiseau serait remboursé, aux frais de justice près, seulement de ce qu'il a payé à Sébastien.

42. Ces faits sont mentionnés dans la lettre d'Amelot à Caze de la Bove du 21 novembre 1781, mais la lettre du 9 septembre n'est pas dans le dossier.

43. Le prix de la pension n'est pas clair. Il est tantôt dit qu'il est de 522 livres, tantôt que ce prix comprend aussi les frais de la maréchaussée. Le prix de la pension pourrait donc être un peu plus faible. 
transféré à La Rossignolerie, où il entre le 11 décembre ${ }^{44}$. Ses ennuis ne s'arrêtent pas là : il avait quitté son régiment de dragons pour s'installer à Guérande; quelques jours après l'avoir conduit à Angers, la maréchaussée de La Roche-Bernard reçoit l'ordre de l'arrêter comme déserteur ${ }^{45}$.

\section{La vente de la seigneurie de Lauvergnac}

Quelques jours après, le 24 décembre 1781, Jean-Sébastien de Silz vend la seigneurie de Lauvergnac. Cette dernière était, au XVII ${ }^{\mathrm{e}}$ siècle, la propriété de Pierre de La Haye de Silz. Elle était alors centrée sur le château de Lauvergnac (aujourd'hui commune de La Turballe), mais elle comprennait des terres au nord de ce château, jusqu'à La Roche-Bernard. À la suite d'un double mariage de Pierre de La Haye de Silz ${ }^{46}$, la seigneurie de Lauvergnac fut divisée en deux parties à partir de 1749, avec d'un côté la seigneurie de Lauvergnac en Piriac et Guérande, centrée sur le château de Lauvergnac, et de l'autre la seigneurie de Lauvergnac en Herbignac et Férel, qui appartient à Jean-Sébastien de La Haye de Silz. Cette seconde seigneurie ne comporte pas de château, mais des terres, un hameau (Lauvergnac, à Herbignac), des maisons et deux moulins.

La vente s'effectue en faveur de Pierre Marie Le Sénéchal de Kerguizé et de son épouse Marie-Geneviève Maudet de Penhouët. La famille Le Sénéchal de Kerguizé, de vieille noblesse bretonne, réside à la belle saison dans son château de Cohanno et passe l'hiver à Vannes. L'acte de vente est signé en l'hôtel de Jean-Sébastien de Silz dans cette ville; il a disparu, mais nous disposons d'un résumé de cette vente chez le notaire et de copies partielles dans d'autres actes. Le prix de vente est de 40000 livres $^{47}$ : d'une part un principal de 6000 livres, qui ne paraît pas mentionné dans l'acte et ne fait l'objet d'aucune quittance, ce qui aura des conséquences importantes; d'autre part une rente viagère de 4000 livres ${ }^{48}$ par an, sur deux têtes, celle de Jean-Sébastien et celle de son épouse Antoinette. Le prix de 40000 livres peut paraître élevé, car il n'y a pas de château. À titre de comparaison, le château de Silz sera vendu en 1797 pour 27000 francs (environ 27000 livres). Mais la seigneurie de Lauvergnac comporte un grand nombre de terres et de maisons, ce qui explique son prix. Accompagné de deux notaires, Pierre Marie Le Sénéchal de Kerguizé entre en possession de la seigneurie le 30 janvier 1782; l'entrée en possession se déroule jusqu'au

44. Arch. dép. du Morbihan, E 5236. Lettre de frère Eunuce à Guibert, 8 décembre1782,

45. Arch. dép. du Morbihan, E 5236. Lettre de Thomas de Bénéac, 6 avril 1783.

46. Arch. dép. du Morbihan, 1 J 556. BAudry, Gilbert, La Seigneurie de Lauvergnac..., op. cit.

47. C'est ce que mentionne le résumé de l'acte, qui parle d'un principal de 6000 livres (Arch. dép. du Morbihan, 6 E 2177). Selon une autre source, un principal de 6535 livres est mentionné (Arch. dép. de Loire-Atlantique, B 10437, Juridiction d'Assérac).

48. Cette rente viagère de 4000 livres par an représente le salaire annuel de 25 journaliers agricoles, si on admet pour ces derniers un salaire annuel de 160 livres. Il s'agit donc d'un revenu important. 
27 mars 1782 et nécessite 36 jours de travail des notaires. Un acte très long et précis de 179 pages décrit les éléments de la seigneurie, maison par maison ${ }^{49}$. Les maisons et les terres se trouvent sur Herbignac, Férel, et La Roche-Bernard.

Pourquoi Jean-Sébastien vend-il Lauvergnac juste après l'incarcération de son fils? Dans son rapport de 1783, Thomas de Bénéac répond que c'est peut-être pour payer les pensions de ses enfants et celle de Sébastien. On peut se demander si ce n'est pas pour assumer un train de vie élevé et si l'enfermement de Sébastien ne favorise pas une vente à laquelle il se serait opposé. La vente de Lauvergnac aggrave le conflit entre le père et le fils.

\section{Les années d'enfermement}

\section{La nouvelle vie de Sébastien}

La Rossignolerie, aujourd'hui lycée David d'Angers à Angers, a été achetée en 1773 par les frères des Écoles chrétiennes. Outre l'institut éducatif, La Rossignolerie comporte une maison de force, avec une soixantaine de détenus de force (dont des aliénés) ${ }^{50}$. L'institution, dont les plans de l'époque nous sont parvenus ${ }^{51}$, a des locaux séparés pour les pensionnaires ordinaires et les détenus de force, qui ont une cour et un réfectoire à part. La chapelle seule réunit les deux publics mais les détenus de force accèdent à une tribune de la chapelle par une entrée séparée et ne sont pas en contact avec les autres pensionnaires.

La vie de Sébastien à La Rossignolerie nous est connue grâce à la correspondance reçue par son avocat $\mathrm{M}^{\mathrm{e}}$ Guibert, gradué en droit et avocat au parlement de Bretagne. Cette correspondance se trouve aujourd'hui dans le fonds Le Sénéchal de Kerguizé des archives départementales du Morbihan ${ }^{52}$. Guibert est aussi l'avocat de $\mathrm{M}^{\mathrm{me}}$ de Kerguizé, puis son chargé d'affaires pour Lauvergnac; révoqué par elle en 1787, il lui a vraisemblablement rendu sa correspondance concernant Lauvergnac, ce qui explique la présence des lettres de Sébastien de Silz dans ce fonds. La correspondance contient 16 lettres écrites par Sébastien de Silz à $\mathrm{M}^{\mathrm{e}}$ Guibert, la première le 17 novembre 1782, la dernière le 11 février $1784^{53}$. D'autres lettres concernant Sébastien et reçues par Guibert sont également dans ce fonds, par exemple une lettre de la marquise du Guesclin ou une lettre du frère

49. Arch. dép. de Loire-Atlantique, $1 \mathrm{~J} 185$.

50. CELLIER, Dominique, Les pensionnats des Frères des Écoles chrétiennes au XVIII siècle, Mémoire de Master 2, Université de Paris IV Sorbonne, 2014, donne de nombreuses indications sur cette institution. Les frères des Écoles chrétiennes tiennent 3 maisons de force en France. La proportion d'aliénés est d'environ 50 \% en 1790. Le prix de la pension des détenus de force est au minimum de 500 livres. Il n'existe pas de liste de détenus.

51. CELLIER, Dominique, Les pensionnats..., op. cit., donne quatre gravures des locaux avant la Révolution.

52. Arch. dép. du Morbihan, E 5235 à E 5245.

53. Les 14 premières lettres, en 1782-83, figurent aux Arch. dép. du Morbihan dans le dossier E 5236, les deux dernières, en 1784, dans le dossier E 5244. 
Eunuce $^{54}$. À La Rossignolerie, Sébastien peut recevoir des visites; il peut également écrire et recevoir des lettres, même si le courrier est préalablement lu. Il a obtenu le droit de gérer ses affaires, certainement après une procédure d'appel contre la décision du 21 janvier 1782 du présidial de Vannes qui en confiait la gestion à son père; dès le mois de juillet 1782 , Guibert est son avocat, et Sébastien lui donne des ordres sur ses affaires.

Sébastien se plaint dans presque toutes ses lettres d'être enfermé contre sa volonté. Il écrit par exemple le 27 juillet 1783 : « Liberté, ma chère liberté; je suis né français et je suis esclave. "Il ne fait pas de reproche particulier à La Rossignolerie et a en bonne estime les frères, notamment le frère Eunuce et le frère Liboire. Au bout de quelques mois, il change de bâtiment à l'intérieur de La Rossignolerie (lettre du 27 février 1783) ${ }^{55}$ :

"Il y a longtemps que j'ai quitté le logement où vous m'aviez vu ${ }^{56}$. Le marquis du Guesclin voulut bien en demander un autre qu'il obtint du Directeur par le bon témoignage que rendit de ma conduite le frère Antoine qui nous dirigeait. Je pouvais me promener toute la journée. Depuis nous sommes dans le nouveau bâtiment et je n'ai pas lieu de me plaindre. Car enfin, je suis prisonnier, je dois paraître coupable et en subir le sort. "

Les pensionnaires, s'ils ont de l'argent, peuvent améliorer leur ordinaire : on peut acheter du bois pour faire du feu; Sébastien (lettre du 12 octobre 1783) évoque la possibilité d'acheter une pièce de vin d'Anjou pour 50 livres. Il décrit ainsi sa vie, non sans humour (lettre du 27 août 1783) :

"Personne n'est aussi sensible que moi, j'en suis honteux mais je suis né ainsi. Comme j'oublie dans le moment ici mes malheurs passés, et que je me flatte de l'espoir de vous voir en peu, je travaille jours et nuits à élever et démolir des châteaux en Espagne, digne et seule occupation d'un grand homme tel que moi. Car quant à l'écriture, on ne s'amuse pas ici à de telles bagatelles. Ce n'est pas l'ordre de la maison : être sage et exact observateur des règles, voilà tout ce que l'on exige, voilà à quoi je m'étudie; aussi, ai-je la consolation de vivre assez tranquille. Dans mon malheureux état, je n'ai qu'à me louer de la manière honnête dont le frère Liboire, notre directeur, a travaillé à faire expédier mes affaires. Je suis redevable à tout le monde. C'est dans mon malheur ma seule consolation. "

Et (lettre du 27 juillet 1783) :

"Au sujet de l'écriture et du chiffre, ce n'est pas la coutume d'enseigner les pensionnaires de force; aussi on ne le fait pas. Quant à la promenade, il n'y a point de distinction; ici, tout le monde sort à certaines heures et rentre de même. Voilà la règle et il faut s'y conformer. "

54. Ces lettres sont dans le dossier Arch. dép. du Morbihan, E 5236.

55. Ce changement de bâtiment correspond en fait à une réorganisation de La Rossignolerie, terminée fin 1782, et évoquée par CELLIER, Dominique, Les pensionnats..., op. cit.

56. Guibert est venu le voir une fois, en 1782. 
La direction de l'établissement, dans un souci d'anonymat des pensionnaires et d'égalité entre eux, les oblige à prendre des pseudonymes. Ils sont appelés et s'appellent entre eux du nom d'un saint; pour simplifier le service du courrier, il est recommandé que le courrier reçu porte le nom de ce saint. Sébastien de Silz s'appelle " saint Félix ${ }^{57}$ "; il signe ses deux dernières lettres ( 8 janvier et 11 février 1784) de ce nom et demande à Guibert de lui envoyer son courrier avec ce nom sur l'enveloppe.

Le frère Eunuce, dans une lettre du 6 décembre 1782 à Guibert, explique que Jean-Sébastien est en retard pour le paiement de la pension. Sébastien se plaint dans ses lettres de l'attitude de son père et de sa belle-mère, sur le plan affectif et financier. Il considère que son père l'a trompé lors de la succession de sa mère, en s'appuyant sur la faiblesse du notaire (lettre du 24 mai 1783). Son père s'est ensuite approprié des récoltes du grenier de Missillac qui devaient lui revenir en partie (lettre du 23 décembre 1783). Il ajoute dans cette dernière lettre :

" De tout ce que ma mère possédait, il n'avait rien fait passer dans l'inventaire que la montre qu'il m'avait donné et qu'il m'a bien fait payer. Il a vendu chez Deguye à Nantes la boëte d'or et le reste pour acheter une toilette d'argent à Madame la Comtesse et voilà comment mon père en a agi à mon égard, ne m'ayant jamais donné un sol, exigeant quittance de moi pour tout ce qu'il me donnait et d'après même mes quittances il ne m'en envoyait pas le montant. A la vérité, $\mathrm{M}^{\mathrm{me}}$ de Silz me marquait qu'on y aurait eu égard, j'en doute. Il ne m'a donné que sept chemises depuis deux ans que je suis renfermé. J'ai été obligé d'en rendre deux que je devais. Resté à cinq. On nous rend le linge que tous les mois d'hiver, aussi je suis forcé à porter des lambeaux. Cela paraît incroiable, mais c'est la vérité.

J'ai eu l'honneur de lui écrire une fois et je lui marquais que deux ans de prison pourraient bien faire oublier mes fautes ou que sinon, j'espérais qu'il ne trouverait pas mauvais que je sollicite auprès du ministre pour obtenir une espèce de liberté sans sortir de la maison où j'étais, ou, si cela était impossible, comme cela l'est, dans un château et que je ne craignais pas de vivre sous les yeux de gens qui sans partialité auraient pu rendre à sa majesté un témoignage de ma façon d'agir. Il ne m'a pas répondu mais je gémis toujours dans l'espoir et jamais je ne vois la fin de mes maux."

Sébastien n'est pas tendre avec sa belle-mère (lettre du 17 décembre 1782) : "Je ne puis pas être plus malheureux que depuis que j'ai une marâtre, car tant que cette charmante femme vivra, mon père pensera toujours de la même façon. " Et dans sa lettre du 4 octobre 1783 : "Vous savez que les de Silz, de tout temps bonne pâte de gens, ont été en femme les plus malheureux de tous les hommes. J'en ai même un exemple de bien frappant devant les yeux."

57. Nous avons contacté à ce propos le service des archives des frères des Écoles chrétiennes à Rome. Le frère Alain Houry nous a confirmé que les pensionnaires de force recevaient à l'époque un nom de saint pour assurer leur anonymat. Les archives de Rome conservent une correspondance entre le frère Liboire et M. Amelot. 
Jean-Sébastien de Silz était en froid avec son frère cadet Honoré, le chevalier de Silz, officier au régiment d'Enghien. Ce dernier avait désapprouvé le remariage de Jean-Sébastien. Selon Sébastien, sa belle-mère avait tout fait, pour réconcilier le chevalier avec son père, en lui permettant notamment de passer l'hiver au château de Silz, alors qu'il passait ses congés d'hiver chez un cousin à Rennes, pour empêcher Sébastien d'en faire un allié (lettre du 17 décembre 1783) :

"Le chevalier de Silz étant réconcilié avec mon père, il ne voudra sûrement pas rompre avec lui et avec $\mathrm{M}^{\mathrm{me}}$ de Silz pour moi ? Je ne le blâme pas, le chevalier doit être fort content d'avoir la maison de mon père pour passer les cartiers d'hiver et je crois pouvoir vous assurer que c'est encor un coup parti de la rusée $\mathrm{M}^{\mathrm{me}}$ de Silz car enfin, quand mon père l'épousa, le chevalier, qui était chez mon oncle de Kerhaude lui écrivit. Mon père ne voulut point le voir et $\mathrm{M}^{\mathrm{me}}$ de Silz ne le sollicita qu'autant que la bienséance l'exigeait. J'étais pour lors à la maison mais maintenant que je suis renfermé et qu'elle ne voit point mes frères qui sont dans la marine, qu'elle a fait mettre mes sœurs au couvent et n'ignorant pas que le chevalier comme frère de mon père pouvait beaucoup et par rapport à moi pour me tirer d'ici et par rapport à elle-même pour empêcher la dissipation qu'elle fait des biens de mon père, elle ne l'a fait venir chez elle que pour en faire ce qu'elle voudra. Si vous voyez mon oncle, il vous dira sûrement le portrait qu'elle lui a fait de moi. Il vous dira aussi qu'il a vu une lettre d'un de mes officiers et une du marquis de Sérent ${ }^{58}$ : je sais ce qu'elles contiennent, je les ai vu avant lui. Je souhaite qu'il s'intéresse à mon sort. "

Sébastien reconnaît des fautes dans son comportement dans une lettre à son père (lettre citée plus haut du 24 mai 1783), et dans d'autres lettres : " J'ai été jeune et étourdi " (lettre du 12 octobre 1783); " Faites paraître aux yeux du ministre ma conduite telle qu'elle a été, soit au régiment, soit en Bretagne. L'une ne fait pas mon éloge mais l'autre parle bien en ma faveur car l'on est toujours repentant lorsqu'on reconnaît sincèrement ses fautes " (lettre du 27 juillet 1783).

Malgré les reproches qu'il lui adresse, Sébastien n'est pas rancunier envers son père (lettre du 12 octobre 1783) :

"Vous me marquez que mon père est indisposé : je l'ignorais, je ne suis pas assez heureux pour recevoir de ses nouvelles. Aussi, le plus grand plaisir que vous puissiez me faire, c'est de m'en donner toutes les fois que vous m'écrirez. Quoique malheureux par son ordre, personne ne l'aime plus sincèrement que moi et ne désire plus ardemment le rétablissement de sa santé. S'il avait voulu croire des gens bien intentionnés, je serais Capitaine de Dragons."

Il hésite même, pour sa défense, à signer lui-même le placet de son avocat et à critiquer publiquement son père (lettre du 6 février 1783) :

58. Armand Louis de Sérent (1736-1822) est maréchal de camp, et à partir de 1780 gouverneur des jeunes ducs d'Angoulême et de Berry. Sa famille est originaire de Sérent, à proximité de Malestroit. 
" Je trouve fort inusité de [m'] avoir fait signer votre placet étant renfermé, je suis sensé ignorer tout ce que peut faire mon père. De plus, je vous avoue que vouloir être soi-même l'accusateur de son père, répugne trop à la nature. Je sais que mon père a bien des torts mais il n'est pas de moi à le juger, c'est à ma famille, qui doit faire le tout pour le mieux. Tâchez d'avoir mon élargissement, voilà ce que je souhaite, d'après cela je suis un Caton capable de supporter tous les malheurs qui peuvent arriver dans la vie, puisque je vis encore et me porte assez bien, moi qui suis depuis quatorze mois privé de ma liberté, de ce trésor de la vie, de ce bien si naturel à tous les hommes nés libres."

Sébastien, dès qu'il a un avocat, organise sa défense pour être libéré et contre-attaque à propos de Lauvergnac. Nous commençons par ce second point.

\section{Contre-attaque à propos de Lauvergnac}

Sébastien s'oppose à la vente de Lauvergnac en entamant une procédure de retrait lignager contre la famille Le Sénéchal de Kerguizé. Le retrait lignager est une procédure d'Ancien Régime par lequel un tiers, le " retrayant ", se substitue à l'acquéreur d'un bien pour se l'approprier. Il permet aux membres d'un lignage de reprendre un bien qui est vendu au membre d'un autre lignage, à condition de rembourser à l'acquéreur le prix d'achat du bien. S’il bénéficie du retrait lignager, Sébastien de Silz pourra reprendre la seigneurie de Lauvergnac, à condition de rembourser la famille de Kerguizé.

La demande de retrait lignager de Sébastien sur Lauvergnac a été précédée d'une demande de $\mathrm{M}$. et $\mathrm{M}^{\text {me }}$ de Rivière, qui sont des cousins des de Silz ${ }^{59}$. Il y a donc deux demandes concurrentes de retrait lignager pour Lauvergnac. M. et $\mathrm{M}^{\mathrm{me}}$ de Rivière, en étudiant l'acte de vente de Lauvergnac, ont constaté que le principal de 6000 livres n'était pas mentionné et qu'on n'évoquait pas de quittance donnée lors de l'achat pour cette somme. La famille de Rivière avance donc que ce principal n'existe pas ou n'a pas été payé (il a sans doute été payé, mais dissimulé pour des raisons fiscales). Comme, juridiquement, le principal est inexistant, il suffit de verser la rente viagère annuelle de 4000 livres pour devenir retrayant de Lauvergnac. Une décision en appel du parlement de Rennes du 4 avril 1782 tranche sur ce point en faveur de la famille de Rivière ${ }^{60}$, sans cependant leur attribuer le retrait lignager, qui fera l'objet d'une décision ultérieure. La demande de retrait lignager de Sébastien de Silz, représenté par Guibert, arrive au tribunal d'Assérac en juillet 1782, en concurrence avec la demande de la famille de Rivière. Le 26 août 1782, le tribunal d'Assérac privilégie la demande de Sébastien, plus proche sur le plan familial; il est maintenant " retrayant "

59. Dans sa lettre du 24 mai 1783, Sébastien parle de sa cousine Madame de Rivière, qui lui a écrit et à laquelle il n'a pas voulu répondre.

60. Arch. dép. du Morbihan, E 5236. 
de Lauvergnac ${ }^{61}$, se fait appeler " Monsieur de Lauvergnac " et signe des lettres sous ce nom.

Compte tenu de la décision précédente du parlement de Rennes, Sébastien n'a pas de principal à rembourser à la famille de Kerguizé; il doit seulement lui verser 4000 livres de rente annuelle, que la famille de Kerguizé reversera à $M$. et $\mathrm{M}^{\mathrm{me}}$ de Silz. Sébastien se retrouve dans une situation singulière : bien qu'enfermé à La Rossignolerie par son père, il finance lui-même la rente viagère de son père et de sa belle-mère. Sébastien compte sur les revenus de Lauvergnac pour payer la famille de Kerguizé; en 1782 et 1783, il perçoit, par l'intermédiaire de Guibert, ces revenus et effectue des reversements à Pierre Marie de Kerguizé, qui lui en donne quittance ${ }^{62}$. Sébastien de Silz ne peut pas payer 4000 livres par an : comme l'indiquent les livres de comptes de Lauvergnac ${ }^{63}$, les recettes de la seigneurie sont d'environ 2000 livres par an. Sébastien, enfermé et sans autre ressource, ne pourra pas payer à la longue. C'est ce qu'a compris la famille Le Sénéchal de Kerguizé, qui, déçue de la décision judiciaire, fait contre mauvaise fortune bon cœur; elle pense que tout ceci se terminera avec la libération de Sébastien, qu'il vaut mieux garder de bonnes relations avec lui et l'aider à être libéré.

\section{Comment sortir de La Rossignolerie?}

Sébastien fait tout ce qu'il peut pour être libéré; son avocat escompte au début une libération rapide, qui ne se produit pas. Sébastien mobilise famille et amis, et intervient auprès de la maison du Roi. Il a sans doute tenté d'impliquer ses frères et sœurs mais ils avaient peu de moyens, étant tous mineurs sauf Antoinette, placée dans un couvent. Auguste de Silz, officier de marine, participe à ce moment à la guerre d'indépendance américaine : il est blessé lors du combat des Saintes en avril $1782^{64}$, emprisonné à La Jamaïque et en Angleterre. Leur père n'entendait pas les arguments de ses enfants.

Dans les lettres reçues par Guibert, Sébastien dit avoir écrit à de nombreux oncles ou cousins plus ou moins proches, son oncle le chevalier de Silz, son oncle de Kerhaude à Rennes, son oncle de Couëssel, son cousin Payen, les cousins de Sérent, d'autres qui sont peut-être seulement des amis, le marquis de Grégo, $\mathrm{M}^{\mathrm{me}}$ de la Bretèche, $\mathrm{M}^{\mathrm{me}}$ de Chalus. Il est également en relation avec le marquis du Guesclin, la marquise du Guesclin, enfin la duchesse de Gesvres (née du Guesclin).

61. Arch. dép. du Morbihan, B 10435, Tribunal d'Assérac, 1781-1782.

62. Ces quittances figurent dans le fonds Le Sénéchal de Kerguizé (Arch. dép. du Morbihan, E 5236).

63. Arch. dép. du Morbihan, E 5235.

64. ANTIER, Jean-Jacques, L'amiral de Grasse vainqueur à la Chesapeake, Éditions maritimes et d'outre-Mer, 1971, p. 165-221, analyse en détail la bataille des Saintes. 
Ces membres de la famille du Guesclin sont probablement des cousins éloignés. Il existe à cette époque encore deux branches de la famille du Guesclin, la branche La Roberie, et la branche de Beaucé. Françoise Marie du Guesclin, née en 1737, a épousé le duc de Gesvres et sera la dernière descendante de la branche La Roberie. L'autre branche, installée à Beaucé en Anjou, a pour dernier descendant Bertrand Henri Michel du Guesclin, né en 1743. Le marquis du Guesclin, dont la marquise du Guesclin est la mère, n'est pas marié; il est brigadier dans les chevau-légers de la garde, maître de camp d'infanterie, et est souvent à Versailles. Si la duchesse de Gesvres ne répond pas aux sollicitations, le marquis du Guesclin est sensible à l'enfermement de Sébastien; il vient souvent le voir à La Rossignolerie, mais il est absent à certains moments et malade. La marquise du Guesclin agit également en faveur de Sébastien : elle envoie auprès de lui diverses personnes, dont l'abbé de Villeneuve, doyen de l'église d'Angers. Ce dernier rend visite une fois à Sébastien puis écrit à Guibert le 7 octobre 1782 qu'il n'ira plus : le lieu n'est pas " attrayant " et " il y a toujours du risque à se mêler des affaires des personnes qui y sont détenues ou par ordre du Roy ou par la volonté de leurs parents".

Une première tentative pour libérer Sébastien a lieu dès la première année. Le frère Eunuce s'en fait l'écho dans une lettre à Guibert (6 décembre 1782), en évoquant une lettre de Jean-Sébastien de Silz : « Il [Jean-Sébastien] ignore le projet qu'a la famille de notre pensionnaire de le retirer à la fin de son année qui sera le 11 courant. " Cette première tentative aboutit début 1783 à l'envoi d'un placet à M. Amelot, signé par Sébastien, avec des lettres de soutien de personnes de sa famille et d'amis; ce placet n'a pas été conservé, mais Thomas de Bénéac donne des éléments sur son contenu dans sa lettre du 6 avril 1783.

Une autre tentative pour le faire libérer se met en place ensuite : elle vise à remettre un placet directement à $\mathrm{M}$. Amelot. Sébastien signe un texte, rédigé par Guibert et envoyé au marquis du Guesclin par l'intermédiaire de sa mère; il écrit à Guibert le 27 février 1783 à propos de ce placet :

" Je l'ai lu et signé le 24 . $\mathrm{M}^{\text {me }}$ [du Guesclin] partait pour Paris le lendemain. Elle veut bien s'en charger et je ne doute point que son fils ne le présente lui-même à $\mathrm{M}^{\mathrm{r}}$ Amelot. Avant sa maladie et son départ pour Paris, il venait me voir deux fois par semaine et m'a toujours promis de s'intéresser pour moi. Aussi j'espère que nous réussirons."

La chance n'est pas du côté de Sébastien : le marquis du Guesclin est très malade et meurt à Paris le 20 mars $1783^{65}$. Le placet ne sera jamais présenté. Toutefois, une autre intervention, de la part de Pierre Marie Le Sénéchal de Kerguizé, a lieu à peu près au même moment; elle a permis de rencontrer M. Amelot et montré que Jean-Sébastien de Silz restait seul maître de la décision de maintenir en détention son fils, qui raconte ainsi les évènements (lettre du 5 avril 1783) :

65. Gazette de France, 25 mars 1783. 
" $\mathrm{M}^{\mathrm{me}}$ la marquise du Guesclin, comme vous le savez, se chargea de mon placet et devait le faire présenter par $\mathrm{M}^{\mathrm{r}}$ son fils. Jugez de la douleur en arrivant à Paris. $\mathrm{M}^{\mathrm{r}}$ se trouve mourant et quelques jours après, l'incroyable mort m'enlève un sincère ami et un respectable protecteur. Vous devez bien penser que dans un si triste moment, $\mathrm{M}^{\text {me }}$ toute accablée à sa douleur, n'a guère pensé à mes affaires et en voilà la preuve : j'ai reçu en date du 28 mars, une lettre de $\mathrm{M}^{\mathrm{r}}$ de Kerguizé qui me marque qu'il a été voir $\mathrm{M}^{\mathrm{r}}$ Amelot avec son parent le Comte de Carcado, lieutenant général des armées du roi, aux fins d'obtenir ma liberté. Telle fut, pour conclusion, la réponse du ministre : la moindre lettre de son père lèvera tous les obstacles et obtiendra sa liberté. $\mathrm{M}^{\mathrm{r}}$ de Kerguizé ne m'en dit pas davantage, si ce n'est qu'il me marque qu'il est sur le point de partir pour Vannes et qu'il fera son possible auprès de mon père. Cela ne m'offre pas une heureuse perspective, toutes vos peines et vos soins ont été en vain : je suis né malheureux, il faut que je subisse mon malheureux sort." "

Sébastien semble ensuite découragé, dépressif. Son écriture est très nerveuse, malhabile (lettre du 27 juillet 1783). Toutefois, il se reprend vite. Après le premier placet, Amelot envoie le 15 mars 1783 une lettre à l'intendant de Bretagne en demandant des explications, car les arguments de Sébastien lui semblent mériter attention :

" C'est, Monsieur, sur votre avis que j'ai expédié le 23 novembre 1781 les ordres du Roi en vertu desquels le sieur de La Haye de Silz a été conduit dans la maison des Écoles chrétiennes d'Angers. Il demande sa liberté par le mémoire que vous trouverez ci-joint. Ses représentations paroissent mériter quelques attentions. Je vous prie de vous en faire rendre compte et de me marquer ce que vous en pensez. "

Le placet de Sébastien est transmis le 17 mars à Thomas de Bénéac. Ce dernier répond le 6 avril par un rapport assez long sur Sébastien et sa famille, déjà évoqué dans la première partie de cet article; il est à charge contre Sébastien et démonte systématiquement tous ses arguments. Thomas de Bénéac remarque que, parmi les personnes qui se sont associées à ce placet, peu sont de sa famille, seulement les Payen, père et fils, alors que Sébastien a à Guérande d'autres gentilshommes de sa famille, M. de Couëssel, M. de la Bourdonnaie, M. de Trévelec, qui ne se sont pas joints à la demande de libération. Thomas de Bénéac nous apprend néanmoins que quatre prêtres, un avocat, deux procureurs, un chirurgien, et d'autres personnes se sont associés au placet : c'est peut-être ce qui a inquiété à Versailles. Il minore systématiquement l'importance de ces soutiens, souligne que les deux procureurs sont du même siège, que certaines personnes ne sont pas de Guérande. Le placet de Sébastien critique les affaires financières et les ventes conduites par son père depuis qu'il est enfermé; au contraire Thomas de Bénéac les défend, justifiant des ventes qu'il ne connaît pas toujours bien. L'une d'elles associe Jean-Sébastien à son frère le chevalier de Silz, dans un remboursement commun de dette : elle explique peut-être pourquoi ce dernier n'est pas intervenu pour soutenir son neveu. En ce qui concerne la vente de Lauvergnac, Thomas de Bénéac affirme que le retrait lignager de Sébastien est une simulation et que 
Le Sénéchal de Kerguizé continue à signer les papiers de Lauvergnac, ce qui est faux $^{66}$. Sébastien critiquait également les avantages consentis à sa bellemère lors du contrat de mariage de 1775, avec un douaire de 20000 livres en faveur d'Antoinette de Silz; cette somme ne paraît pas exagérée à Thomas de Bénéac. Ce dernier conclut que le mémoire de Sébastien est faux ou exagéré. Les pièces remises par son père en 1781 "manifestent le danger qu'il y auroit à lui rendre sa liberté ". Sur la base de ce rapport, l'intendant de Bretagne conclut la lettre qu'il envoie à M. Amelot en affirmant : " les représentations du sieur Delahaye ne méritent aucun égard ". Sébastien reste emprisonné.

La libération semble maintenant devoir venir de Bretagne. Des interventions sont faites auprès du père de Sébastien. Sébastien, qui pense que l'évêque de Vannes a participé à la demande de lettre de cachet, charge Guibert d'agir auprès de lui (lettre du 22 octobre 1783$)^{67}$ et lui demande aussi d'aller voir M. de Kerguizé pour qu'il intervienne à nouveau. Mais Jean-Sébastien de Silz ne veut pas que son fils sorte de La Rossignolerie. D'autres raisons feront retrouver la liberté à Sébastien.

\section{La sortie de La Rossignolerie}

Les relations de Sébastien avec son avocat et avec la famille de Kerguizé se dégradent. Il est libéré en 1785 et Lauvergnac reste à la famille de Kerguizé. Jean-Sébastien meurt quelques années plus tard, en 1791.

\section{Les relations de Sébastien de Silz avec $M^{e}$ Guibert et avec la famille de Kerguizé}

Dans sa lettre du 11 février 1784 à Guibert, la dernière dont nous disposons, Sébastien s'étonne que son avocat ne lui ait pas rendu compte de l'entrevue qu'il a eue avec son père; il veut faire un nouveau placet, par l'intermédiaire de Guibert. Son cousin de Trévelec lui a écrit, il lui a dit que lui et d'autres parents le soutenaient, comme la comtesse de Francheville; que son oncle le chevalier de Silz l'aimait beaucoup et serait prêt à faire quelque chose pour lui. Pour cette nouvelle tentative, Guibert devait contacter le comte de Goyon, lieutenant général de la province, la duchesse de Gesvres ou la duchesse de Lorge (dont il dit qu'elle est sa parente) pour qu'ils présentent le placet au baron de Breteuil, nouveau secrétaire à la maison du Roi depuis l'automne 1783. Guibert, avec qui Sébastien a jusque-là de bonnes relations personnelles, n'est pas très actif. Né en 1710, il a 74 ans en 1784 et traite les affaires de Sébastien avec une certaine distance. Par ailleurs, il est aussi depuis longtemps le chargé d'affaires de $\mathrm{M}^{\mathrm{me}}$ de Kerguizé.

66. Les archives de la famille Le Sénéchal montrent au contraire que les revenus de Lauvergnac sont versés au nom de Sébastien et non à celui de Pierre Le Sénéchal.

67. Monseigneur Sébastien Michel Amelot (1741-1829), évêque de Vannes depuis 1775, est un cousin éloigné d'Antoine Jean Amelot. 
Il est dans une situation délicate vis-à-vis de Sébastien, à mesure que les relations de celui-ci se tendent avec la famille de Kerguizé. Sébastien a sans doute changé d'avocat au cours de l'année $1784^{68}$, ce qui expliquerait l'arrêt de sa correspondance avec Guibert.

Le 10 février $1784^{69}$, Pierre Marie Le Sénéchal de Kerguizé reconnaît avoir reçu de Guibert 1000 livres provenant de "Monsieur de Silz de Lauvergnac ". Sébastien est toujours " retrayant " pour Lauvergnac mais il ne peut verser l'intégralité des 4000 livres de rente viagère à la famille de Kerguizé. M. de Kerguizé l'a assigné par deux fois, le 19 août 1783, puis le 16 avril 1784, devant le tribunal d'Assérac. Le 30 août 1784 le tribunal condamne Sébastien de Silz à payer à M. de Kerguizé 1490 livres en retard ${ }^{70}$.

Guibert, chargé d'affaires de $\mathrm{M}^{\mathrm{me}}$ de Kerguizé, lui donne désormais des conseils à propos de Sébastien. Dans une lettre du 24 octobre $1784^{71}$, il lui dit :

" J'ai donné avis à M. de Kerguizé de la dernière lettre de M. de Lauvergnac, et ai aussitôt répondu à cette lettre comme je le devais faire. Je pense qu'il y réfléchira. Ce qui le fait chanceler, c'est qu'on lui promette son élargissement pour mieux le surprendre. "

Guibert juge la stratégie de M. de Kerguizé vis-à-vis de Sébastien « bien cimentée ". M. de Kerguizé tente-t-il d'échanger son soutien à Sébastien contre un désistement de Lauvergnac?

\section{La libération de Sébastien de Silz}

La libération de Sébastien est liée à deux décisions politiques. La première est essentielle : elle s'exprime dans une circulaire du 25 octobre 1784 du baron de Breteuil, qui a remplacé $\mathrm{M}$. Amelot à la tête de la maison du Roi. La circulaire Breteuil prévoit que, pour les lettres de cachet au motif de prodigalité ou débauche, la durée de détention ne puisse pas dépasser deux ans. Le baron de Breteuil exige que l'intendant ou ses subdélégués se déplacent chaque année dans les maisons d'enfermement pour recueillir des informations sur les détenus ${ }^{72}$. La seconde décision est l'ordonnance royale du 17 décembre 1784 " portant amnistie générale en faveur des soldats, cavaliers, hussards, dragons et chasseurs qui ont déserté des troupes de Sa Majesté avant le $1^{\mathrm{er}}$ janvier 1785 ». L'ordonnance prévoit que les commandants de troupes sont autorisés à admettre dans leurs régiments les déserteurs qui, ayant profité de l'amnistie, se présenteront pour y servir.

En application de la circulaire Breteuil, chaque intendant doit remettre un état des détenus par lettre de cachet de sa généralité, avec un avis sur

68. Guibert est encore son avocat en août 1784, devant le tribunal d'Assérac.

69. Arch. dép. du Morbihan, E5244.

70. Arch. dép. de Loire-Atlantique, B 10436.

71. Arch. dép. du Morbihan, B 5244.

72. HAUTEBERT, L'internement..., op. cit., p. 62. La circulaire du baron de Breteuil est aux Arch. dép. d'Ille-et-Vilaine, C 161. 
leur comportement. L'intendant de Tours (dont dépend Angers), Daine, envoie le 26 décembre 1784 une liste au nouvel intendant de Bretagne, Bertrand de Molleville; douze détenus bretons de la maison d'Angers y figurent. Une observation est faite sur chacun, "par une personne sûre ", dit l'intendant de Tours. Trois observations sur douze sont favorables à une libération ${ }^{73}$. Il est dit de Sébastien : "Âgé d'environ 30 ans, cy-devant étourdy, à présent tranquille, paraissant avoir l'esprit et le caractère bon : il est du nombre de ceux qu'il convient de mettre en liberté. "

Le document parvient au baron de Breteuil, qui écrit le 31 décembre à l'intendant de Bretagne. Il propose, pour Sébastien ${ }^{74}$, "de lui rendre la liberté dont il y a lieu d'espérer qu'il fera un bon usage à l'avenir, les motifs qui ont déterminé à l'emprisonner ne portant que sur des faits d'inconduite, de débauche et de dépravation dont il me paroit suffisamment puni par une détention qui dure depuis plus de 3 ans ". Le motif de désertion n'est pas évoqué par le baron de Breteuil et ne paraît pas avoir joué de rôle.

Après approbation de l'intendant de Bretagne, le baron de Breteuil donne l'ordre de libération le 6 janvier 1785 : "Vous trouverez ci-joint, Monsieur, les ordres que vous m'avez proposés pour la liberté du sieur de La Haye de Silz détenu dans la Maison des Écoles Chrétiennes d'Angers. Je vous prie de les faire exécuter. " L'intendant de Bretagne transmet le 12 janvier l'ordre à l'intendant de Tours et confirme à Versailles avoir transmis. L'intendant de Tours répondra le 5 mars avoir fait libérer le 21 janvier trois détenus bretons de la maison d'Angers, et transmet le récépissé signé par frère Liboire : "Le greffier à la subdélégation d'Angers m'a remis l'ordre du Roy pour remettre en liberté le sieur de La Haye de Silz détenu dans notre maison, auquel ordre je m'oblige de me conformer. Fait à Angers le 21 janvier 1785. Frère Liboire. "

Nous ne savons pas quelle a été la vie de Sébastien entre 1785 et 1790 , s'il a tenté de revenir à la Ferme des devoirs ou bien de retrouver du service dans l'armée; en 1793 il fait partie de la Garde nationale de Guérande ${ }^{75}$.

\section{Qu'advient-il de Lauvergnac?}

M. de Kerguizé meurt au début de $1785 . M^{\text {me }}$ de Kerguizé gère désormais Lauvergnac, avec l'aide de Guibert. Celui-ci semble ignorer jusqu'à la fin de 1785 la libération de Sébastien. Dans une lettre du 23 septembre 1785 à $\mathrm{M}^{\text {me }}$ de Kerguizé, il affirme que Jean-Sébastien de Silz, qui avait refusé de remettre les titres de Lauvergnac à son fils ${ }^{76}$, vient de les remettre à $M^{\text {me }}$ de Kerguizé. Dans la lettre du 29 octobre 1785, Guibert, parlant de Sébastien, conseille à $\mathrm{M}^{\mathrm{me}}$ de Kerguizé de " se méfier des chiens enragés ". Les pro-

73. Arch. dép. d'Ille-et-Vilaine, C 158.

74. Arch. dép. d'Ille-et-Vilaine, C 161. Dossier Sébastien de La Haye de Silz.

75. Arch. dép. de Loire-Atlantique, L 1477, et Association Nantes HistorRe, L'insurrection de mars 1793 en Loire-Inférieure, 1993, p. 11.

76. Sébastien lui a fait écrire à ce propos en 1783 par l'intermédiaire de Guibert. 
cédures judiciaires continuent entre Sébastien et la famille de Kerguizé. Dans une lettre du 8 décembre 1785, Guibert avise $M^{\text {me }}$ de Kerguizé, que M. de Silz père, " bien mal conseillé " veut assigner son fils à propos de Lauvergnac. Guibert nous apprend dans sa lettre à $\mathbf{M}^{\text {me }}$ de Kerguizé du 18 décembre 1785 que Sébastien s'est enfin désisté.

La famille de Kerguizé voit ses droits sur Lauvergnac confortés du fait de ce désistement, mais elle n'en a pas fini avec la famille de Silz. En 1787 $\mathrm{M}^{\mathrm{me}}$ de Kerguizé a pris l'avocat Thomas du Cordic comme chargé d'affaires, en remplacement de Guibert. En 1787, elle lui écrit ${ }^{77}$ pour lui demander de régler ses affaires avec les de Silz. Elle doit à Jean-Sébastien de Silz 2500 livres; mais elle se plaint que ce dernier n'a jamais voulu lui payer les 543 livres que lui doit Sébastien. Les 17 juin et 20 juillet 1788, et encore le 2 janvier 1789, elle écrit devoir de l'argent à M. de Silz.

En $1789, \mathrm{M}^{\text {me }}$ de Kerguizé se dit prête à vendre le domaine de Lauvergnac, pour seulement 26000 livres; elle a trouvé un acheteur, mais celui-ci se désiste finalement. En juillet 1789, elle envoie 1602 livres à M. de Silz et demande à Thomas du Cordic de le faire patienter pour les 398 livres impayées de la rente semestrielle, affirmant : "M. de Silz est toujours pressé. " En août 1790, elle reçoit une lettre de $\mathrm{M}^{\text {me }}$ de Silz, qui se plaint de ne pas avoir été payée et qui lui indique que M. de Silz est malade. En septembre 1790, ce dernier écrit lui-même à M ${ }^{\text {me }}$ de Kerguizé : il n'a pas été payé, et a besoin de son argent. Le 15 octobre $1790, \mathrm{M}^{\mathrm{me}}$ de Kerguizé dit ne pas pouvoir payer la rente de M. de Silz; le meunier, et d'autres, ne versent plus ce qu'ils doivent, et elle va devoir emprunter. En 1791, elle a toujours du mal à payer : elle écrit le 29 mai 1791 que M. de Silz la menace d'un procès s'il n'a pas son argent le $1^{\text {er }}$ juillet ${ }^{78}$.

L'achat de Lauvergnac ne s'avère pas une bonne affaire. Fin 1791, les Kerguizé auront payé à Jean-Sébastien et son épouse 10 ans de rente, soit 40000 livres, plus un principal de 6000 livres, 46000 livres au total.

\section{Le décès de Jean-Sébastien}

Jean-Sébastien décède le 13 juin 1791 en son château de Silz; Sébastien devient comte de Silz. Le décès du père renforce la position des enfants visà-vis de leur belle-mère. Trois enfants sont émancipés durant l'été $1791^{79}$.

Le 2 août 1791 commence un inventaire des meubles du château de Silz, en présence de plusieurs enfants, qui déclarent habiter au château. L'inventaire dure six journées complètes, jusqu'au 23 août 1791. L'inventaire de l'argenterie et des bijoux est conflictuel; les relations des enfants avec leur belle-mère ne sont pas apaisées. Le 23 août, Antoinette de Silz accuse

77. Toutes les lettres évoquées dans ce paragraphe proviennent des Arch. dép. du Morbihan, E 3035, Fonds Thomas du Cordic.

78. Il s'agit de la dernière lettre de $\mathrm{M}^{\mathrm{me}}$ de Kerguizé dans le dossier E 3035 des Arch. dép. du Morbihan.

79. Arch. dép. du Morbihan, 17 C 3083, notaire Gillot. 
sa belle-mère, qui a quitté le château 15 jours après le décès de son mari, d'avoir emporté avec elle des bijoux de Marie Élie et une partie de l'argenterie (notamment 16 plats en argent). Elle l'accuse également d'avoir fait enlever du château une valise de vêtements par son frère cadet de Cramezel, avant même le décès de Jean-Sébastien ${ }^{80}$. L'ensemble est noté par écrit dans l'acte d'inventaire. Benjamin Thomas de La Borde répond fermement à Antoinette, en tant qu'avocat de sa belle-mère ${ }^{81}$.

Le 24 août, les comptes de la communauté entre Jean-Sébastien de Silz et Antoinette de Cramezel sont soldés. Du 29 août au 7 septembre 1791, les enfants réalisent une vente publique de meubles au château de Silz.

Une négociation a commencé en juillet, par avocats interposés, entre les enfants de Silz et leur belle-mère à propos de la rente viagère de Lauvergnac. La rente a pour origine la vente de biens appartenant à leur père, dont les enfants s'estiment privés. Ils voudraient récupérer la moitié de la rente mais Antoinette de Cramezel dit avoir contribué pour des sommes importantes à la communauté avec son mari, sommes au moins équivalentes à la rente perçue. Elle a sans doute de bons arguments juridiques, car les enfants reconnaissent le 28 août 1791 son droit à continuer à percevoir la rente de 4000 livres $^{82}$. La seule concession faite est d'opérer sur cette somme un reversement annuel de 150 livres en faveur de Sébastien de Silz. Selon l'acte notarié, cette rente de 150 livres est, de la part de la veuve de La Haye de Silz et envers Sébastien, « une preuve de l'attachement particulier qu'elle a conçu pour lui ". Presque tous les enfants signent l'acte; la veuve de Silz est représentée par Thomas de La Borde. Auguste de Silz est absent de toutes les opérations de l'été 1791 : il a été arrêté à Brest le 22 juin 1791, à bord du Duguay-Trouin, et est détenu durant trois mois et demi à Brest pour des propos jugés contre-révolutionnaires ${ }^{83}$. Une nouvelle partie de la vie des enfants de Jean-Sébastien de Silz a commencé.

Un éclairage nouveau a été porté sur la jeunesse de Sébastien de Silz et sur un conflit avec son père dont a résulté un long enfermement. Sébastien, dont la jeunesse est difficile, est victime de la dureté de son père et d'une situation familiale compliquée. Le sort de ses sœurs au couvent ne paraît pas non plus heureux. Sensible et intelligent, Sébastien est aussi fier et volontaire. S'il n'est pas rancunier envers son père, il ne s'est jamais laissé faire au cours de son enfermement, a constamment lutté pour être libéré. Ses années de détention l'ont endurci, renforcé psychologiquement.

80. Antoinette de Cramezel a deux frères cadets, dont l'un est Jacques de Cramezel.

81. Il épousera l'année suivante Eugénie Armande de Silz et deviendra ainsi le beaufrère d'Antoinette.

82. Arch. dép. du Morbihan, 6 E 10381, notaire Fricot, pour tout ce paragraphe.

83. Arch. nat., F/7/160, Auguste de La Haye de Silz. Selon LevoT, Prosper, Histoire de la ville et du port de Brest, Paris, 1866, t. 3, p. 274-275, il est reproché à Auguste des commentaires désobligeants sur l'aumônier du navire, prêtre jureur. 
Marqué peut-être par le remariage de son père, qu'il estime désastreux, il ne se mariera jamais. À La Rossignolerie, Sébastien comprenait que sa vie ne serait pas facile à sa sortie (lettre du 12 octobre 1783) :

"Mais pouvez-vous croire que je puisse chercher à m'établir en sortant d'une maison de force? Quelle opinion peut-on avoir de moi dans le monde? Les mieux intentionnés me feront la grâce de me regarder comme un étourdi et d'autres comme un libertin : voilà de beaux titres et quel sera celui qui voudra se charger de me présenter chez une femme? Ce ne peutêtre que moi qui par une conduite soutenue et décidée sera dans le cas de faire oublier que j'ai été jeune et étourdi. C'est ce que je désire établir aux yeux de ma province si sa Majesté daigne m'en accorder les moyens, c'està-dire [mon] élargissement. "

La carrière de Sébastien, compte tenu de l'épisode de 1781-1785, n'a pas été brillante lorsqu'éclate la Révolution. Sébastien est désireux de se racheter ou se rattraper, de montrer ses véritables capacités à toute la Bretagne, au service du roi. Ceci contribue à expliquer ses engagements ultérieurs.

On pourrait comparer l'enfermement de Sébastien de Silz à celui d'Honoré Gabriel Riqueti, comte de Mirabeau, futur tribun de la Révolution. Ce dernier a été enfermé à peu près à la même époque par son père, le marquis de Mirabeau, également très dur avec son fils. Le comte de Mirabeau ${ }^{84}$ en vint, à partir de son cas personnel, à une mise en cause des lettres de cachet et de l'arbitraire de la société d'Ancien Régime. Sébastien regrette son emprisonnement, déplore la perte de sa liberté mais ceci ne débouche pas sur une critique sociale de l'enfermement, du système des lettres de cachet et de la société dans laquelle il se trouve.

Nous avons suivi Sébastien de Silz jusqu'en 1791. Une nouvelle vie commence alors pour lui, ainsi que pour d'autres membres de sa famille; elle se termine pour Sébastien par la mort au combat le 28 mai 1795 au hameau de Kervehein à Grandchamp ${ }^{85}$. Cette autre vie mériterait aussi d'être racontée.

84. MirabeAu, Des lettres de cachet et des prisons d'État, Hambourg, 1782. 85. LE FALHER, Jules, Le royaume de Bignan, p. 455, Laffitte reprints, 1994. 


\section{RÉSUMÉ}

Cet article retrace la vie de Sébastien de la Haye de Silz et de sa famille jusqu'en 1791. Avant d'être chef de la Chouannerie du Morbihan en 1794-1795 et de diriger à ce titre des guerriers redoutables comme Cadoudal, Guillemot et Jean Jan, Sébastien a eu une jeunesse tumultueuse, que nous relions au décès de sa mère et au remariage de son père Jean-Sébastien, comte de Silz. Un conflit familial s'engage entre Sébastien et son père, qui demande l'enfermement de son fils par lettre de cachet. À l'issue d'une procédure compliquée impliquant l'intendance de Bretagne à Rennes et la maison du roi à Versailles, Sébastien est enfermé de 1781 à 1785 à la maison de La Rossignolerie d'Angers. Seize lettres de lui nous renseignent sur sa vie en prison. Il s'oppose tout du long à sa détention, en mobilisant tout un réseau, famille, amis et connaissances. La lettre de cachet dont il fait l'objet, et sa défense en réponse (que nous connaissons par ses lettres), apportent un éclairage sur la société d'ancien régime et sur la noblesse bretonne à la veille de la Révolution.

\section{ABSTRACT}

This article examines the life of Sébastien de la Haye de Silz and of his family until 1791. Before becoming leader of the Morbihan Chouannerie in 1794-1795 and commanding formidable warriors like Cadoudal, Guillemot and Jean Jan, Sébastien had a tumultuous youth that can be linked to his mother's death and to the remarriage of his father Jean-Sébastien, count of Silz. A family conflict began between Sébastien and his father in which the latter asked for the imprisonment of his son by lettre de cachet. After a complicated procedure involving the Breton intendant in Rennes and the house of the king in Versailles, Sébastien was imprisoned from 1781 until 1785 at La Rossignolerie in Angers. Sixteen letters written by Sébastien allow us to understand his life in jail. There, he rebelled against his detention and enlisted the help of a whole network of family, friends and acquaintances. The lettre de cachet and his defence, known thanks to his letters, throw light on Ancien Régime society and the nobility of Brittany on the eve of French Revolution. 